\title{
TCS Theory - Planetary Mechanics
}

\author{
Daniel Eduardo Caminoa Lizarralde \\ 78 Herrera y Guzman Street, Cordoba X5008CZB, Argentina \\ Tel: 54-0351-477-0533Ｅ-mail: dcaminoa@gmail.com
}

Received: July 14, 2011 Accepted: August 8, $2011 \quad$ Published: November 1, 2011

doi:10.5539/apr.v3n2p89

URL: http://dx.doi.org/10.5539/apr.v3n2p89

\begin{abstract}
This work continues and expands of the paper The Three-dimensional Complex Space theory - TCS Theory (Caminoa Lizarralde Daniel E, 2010) published in 2010, and it will introduce into the laws that govern all planetary systems, specifically in the domain of global stability and satellite's foundations throughout ours planetary system as part or not of all the galaxies in our cosmos. The most significant finding and certainly surprising, published in this paper at first, refers to the significant implications of solar drag in planetary stability, which is conceptually very different from A. Einstein's deformation field's idea published in General Relativity (Einstein Albert, 1915). Another phenomenon also published at first time in this work and also very significant, is repulsive the electro-gravitational action force between planetary objects that deters and prevents possible collisions between them. It is perfectly clear the inaccuracy of planetary masses considered furthest from the Sun with densities close to the water that occurs because the oldest Newton equation has limited applicability. We make some allusions to galactic and globular cluster's stability, but this issue will be expanded and included in a forthcoming work about the laws on galactic subspace.
\end{abstract}

Keywords: Atomic particles, atomic quarks, Drag of the planetary field, Drag of the sun constant, Electro-gravitational constant, Magneto-gravitational constant, Gravitational constant, Newton equation, Physical constants, Physical space, Planetary quarks, Subspaces

\section{Orbital paths}

The movement of any object in close orbit around another body always responds to an elliptical helix, in some cases the section may look like a circle but it never becomes so. The central body in which turns the orbiting object is always the focus of the hypothetical ecliptic plane closest to the center of the helix turn. This property is general and fundamentally valid, both for atomic mechanics to planetary and stellar mechanics.

It is incorrect to think of a "plane of orbit" as reality is only a virtual image of the cut section of the helix that describes the object in orbit. This concept is of primary importance as we shall see, to understand the forces involved in the stability of the objects in orbit.

\section{Fundamental forces in planetary orbits}

The forces that determine the characteristics of movement throughout planetary orbit are caused by the interaction between planetary quarks and planetary field, these quarks make up every atom of matter, very similar to that happens in the stability of the atom but with interactions with different quarks and within another subspace, see TCS Theory (Caminoa Lizarralde Daniel E, 2010). These interactions are two: the electro-gravitational interaction between the planetary quarks that determine repulsive forces (equal and the same way as the Coulomb force between electrons in the atom) and magneto-gravitational interaction of quarks with the planetary field that determine forces with effective attraction between objects in orbit, this effect is caused by rotation about its axis of the body that acts as a axis or central orbit, is the Sun to the planets orbiting around it -is the Earth for the Moon or artificial satellites orbiting around it- (a vector field is determined by the motion of the quarks of similar action to that described by the Biot's Law (Jean Baptiste Biot, 1820) on the phenomenon of electromagnetic field).

The most significant difference between the gravitational interactions on a global level with regard to electrical and magnetic interactions at the atomic level are: the interactions of electro-gravitational and magneto-gravitational fields that have a broader scope than the interactions that dominate the atomic equilibrium -electric Coulomb's interaction (Coulomb Charles Augustin, 1785) and the interaction of electromagnetic field 
(Jean Baptiste Biot, 1820)-.

But the fundamental phenomenon of attraction between planetary bodies which is due to remain in orbit for any object, is primarily determined by the drag force of the planetary field toward the barycenter of the body of any rotating object directly proportional to the planetary quark content or as in this work we do in relation to their mass. This phenomenon can be observed in rotating bodies with very small mass, gyroscopes, wheel bicycles or cars, etc.

In short, to understand the planetary orbital mechanics we have to analyze the phenomena that occur within the planetary subspace due to the movement of bodies, these phenomena are within TCS Theory (Caminoa Lizarralde Daniel E, 2010): the phenomenon of drag of the planetary field, the electro-gravitational phenomenon, magneto-gravitational phenomenon, the phenomenon of oscillatory magneto-gravitational field and the characteristics of the planetary field around any planetary system.

\section{Drag of planetary field}

The Earth drags the electromagnetic field of the atomic subspace and drag in the same way that the planetary field of the planetary subspace, this happens in all planetary objects in motion. Both drags differ only in one aspect, in the case of electro-magnetic field it travels with the Earth because it is a broken symmetry of the atomic subspace in the periphery thereof, while in the case of planetary subspace will produce this symmetry breaking and the planetary quarks slipped with some resistance to its movement by dragging the planetary field behind it, as if it were a particle moving through a viscous liquid.

To understand the dragging mechanism of the planetary field, we attach the elastic properties of planetary subspace and we studied it by Hooke's Law (Hooke Robert, 1678), with that object we must determine essentially the drag constant of the planetary field where we call here as "drag of the Sun constant" of planetary subspace and by its intimate relationship with Young's modulus (Young Thomas, 1845) identified with the symbol " $Y_{P}$ ".

We do the calculation of this constant from the equation of global stability that will be discussed later, for which now we make the law that govern the drag. The force determined by the drag of the planetary field is directly proportional to the number of planetary quarks dragged, their speed and the constant of the Sun's drag on the planetary subspace. This force can be expressed in terms of the mass of the rotating object, because there is a direct relationship between the number of quarks and the mass. Then we can write to our Sun (subscript " 0 "), with its mass expressed as volume density and any planet (subscript “ $x$ "):

$$
F_{A}=Y_{P} V_{0} \rho_{0} \omega_{0} r_{0} \frac{S_{x}}{d_{A x}^{2}}
$$

where $F_{A}$ is the drag force of the field in Newton, $Y_{P}$ is the drag of the Sun constant global subspace, $V_{0}$ is the volume of the Sun interacts with the planet " $x, \rho_{0}$ is the density of the Sun, $\omega_{0}$ is the Sun's rotational speed expressed as angular velocity, $r_{0}$ is the equatorial radius of the Sun, $S_{x}$ is the surface of the frontal area of planet " $x$ " and $d_{A x}$ is the drag distance to consider between the Sun and the planet.

Solar drag is a planetary subspace phenomenon whose intensity decreases towards the poles of the Sun in the form of infinite parallel planes to the equatorial plane thereof. The mass that corresponds in thickness interacts with the considered planet diameter, as shown in the Figure No. 1.

In the Figure No. 1 shows a planet and its position according to the Sun shown in the figure the Sun's volume " $V_{0}$ " which interacts with the planet -the Sun's hatched area- is displaced in this example but in a parallel position with respect to the solar equatorial plane, with the same movement that show the planet. This shift is a function of bank angle " $\phi$ " measured between the straight line distance between barycenters of the Sun " $0_{0}$ " and the planet " $0 x$ ", and the Sun's equatorial plane, this angle varies from zero -at perihelion and aphelion- up that is equal to the inclination of the orbit planet from the Sun's equatorial plane. Also in this case should note that the distance to be considered in the calculation " $d_{A x}$ " is between centers " $0_{0 x}$ " and the barycenters " $O_{x}$ ", and not precisely in the distance $d_{x}$ that is between barycenters from " $0_{0}$ " to " $0_{x}$ ".

The volume of the Sun interacts with the planet -the hatched area- with reference to Figure No. 1, when the point " 0 " "of the orbit does not belong to the Sun's equatorial plane can be calculated using the following equation.

$$
V_{0}=\frac{\pi h_{2}}{6}\left(3 r_{0}-h_{2}\right)-\frac{\pi h_{1}}{6}\left(3 r_{0}-h_{1}\right)=\frac{\pi\left(h_{1}-h_{2}\right)}{6}\left(h_{1}+h_{2}-3 r_{0}\right)
$$


In the following calculations of this work we only considered variables of the planetary orbits for two points in the same: the perihelion and aphelion, which always belong to the equatorial plane of the Sun. After finding themselves in this case the hatched area apart from Figure No. 1, centered on the Sun equatorial plane, the polar distance " $h_{x}$ " is in perihelion and aphelion, the Sun equal to the radius $r_{0}$ minus the radius of planet " $r_{x}$ ". Because of the latter the equation turns out to be as follows.

Outside the Sun's equatorial plane

$$
\begin{gathered}
\text { being } h_{1}=r_{0}-r_{x}-d_{x} \operatorname{Sin}\left[\phi_{x}\right] \text { and } h_{2}=r_{0}+r_{x}-d_{x} \operatorname{Sin}\left[\phi_{x}\right] \\
\text { then } V_{0 x}=\frac{\pi r_{x}}{3}\left(2 d_{x} \operatorname{Sin}\left[\phi_{x}\right]+r_{0}\right),
\end{gathered}
$$

On the Sun's equatorial plane

$$
\text { being } \operatorname{Sin}\left[\phi_{x}\right]=0 \quad \text { then } \quad V_{0}=\frac{\pi r_{0} r_{x}}{3},
$$

where " $r_{0}$ " is the radius of the Sun, " $r_{x}$ " is the radius of the planet " $"$ " and " $\phi_{x}$ " is the inclination of the straight line joining the barycenters of the Sun and the planet " ${ }_{x}$ " to the equatorial plane of the Sun.

The drag force of the Sun " $F_{A x}$ " on any point of the orbit of a planet can be calculated using one of the following equations. Outside the Sun's equatorial plane is:

being $F_{A x}=Y_{P} \rho_{0} \omega_{0} r_{0} \frac{V_{0} S_{x}}{d_{A x}^{2}} \quad$ and $\quad S_{x}=2 \pi r_{x}^{2} \operatorname{Cos}\left[\theta_{x}\right]$

$$
\text { then } \begin{aligned}
F_{A x}= & Y_{P} \rho_{0} \omega_{0} r_{0} \frac{\frac{\pi r_{x}}{3}\left(2 d_{x} \operatorname{Sin}\left[\phi_{x}\right]+r_{0}\right) 2 \pi r_{x}^{2} \operatorname{Cos}\left[\theta_{x}\right]}{d_{x}^{2} \operatorname{Cos}\left[\phi_{x}\right]^{2}} \\
\therefore \quad F_{A x} & =Y_{P} \rho_{0} \omega_{0} \frac{\pi^{2} r_{0} r_{x}^{3}}{3 d_{x}^{2}}\left(\operatorname{Cos}\left[\theta_{x}\right] \operatorname{Sec}\left[\phi_{x}\right]^{2}\left(2 d_{x} \operatorname{Sin}\left[\phi_{x}\right]+r_{0}\right)\right),
\end{aligned}
$$

being $\operatorname{Cos}\left[\theta_{\mathrm{x}}\right]$ and $\operatorname{Sec}\left[\phi_{\mathrm{x}}\right]$ equal to the unit and $\operatorname{Sin}\left[\phi_{\mathrm{x}}\right]$ equal to zero, in the aphelion and the perihelion on the Sun equatorial plane is:

$$
\begin{aligned}
& F_{A x}=Y_{P} \rho_{0} \omega_{0} \frac{\pi^{2} r_{0} r_{x}^{3}}{3 d_{x}^{2}}\left(\operatorname{Cos}\left[\theta_{x}\right] \operatorname{Sec}\left[\phi_{x}\right]^{2}\left(2 d_{x} \operatorname{Sin}\left[\phi_{x}\right]+r_{0}\right)\right) \\
& \text { being } \operatorname{Cos}\left[\theta_{x}\right]=1, \operatorname{Sec}\left[\phi_{x}\right]=1 \text { and } \operatorname{Sin}\left[\phi_{x}\right]=0 \\
& \text { then } \quad F_{A x}=Y_{P} \rho_{0} \omega_{0} \frac{\pi^{2} r_{0}^{2} r_{x}^{3}}{3 d_{x}^{2}},
\end{aligned}
$$

where " $\phi_{x}$ " is the bank angle of the planet position in orbit at the point to consider with respect to the equatorial plane of the Sun as seen above - do not to be confused with the angle " $\theta_{x}$ " of inclination of the planet orbit to the Sun's equatorial plane-, the other variables have the same references as those given for the above equations.

Because the mass distribution in spherical shape of every star or planet, the maximum intensity of the effects of drag field is manifested on the equatorial plane of the Sun or Earth, with much greater intensity than in the parallel planes near the poles of rotating body, one can see the shape of equipotential field in the Figure No. 2. This difference in density in the dragged field determines that any planetary orbit is elliptical in shape, both planets, as in satellites and a body in orbit. The variation in the density of the field toward the poles, determines an increase in the eccentricity of the virtual plane of the orbit to the extent that increases the inclination or angle to the rotating body equator.

Important note: This algebraic solution of the solar drag phenomenon is a mathematical model that gives correct results. The definitive solution using the infinitesimal calculus will be published in the future after completion of the analysis of the Quantum Electro-Dynamics planetary "QEDp Theory".

\section{Electro-gravitational force}


This planetary force is equivalent to the Coulomb force (Coulomb Charles Augustin, 1785), but as shown in the Figure No. 3, with a sense of repulsion between planetary objects that interact, in addition to this force is independent of the motion to hold the objects under consideration.

Its magnitude " $F_{G E}$ " depends on the interacting objects' masses that are " $m_{\text {Sun }}$ " and " $m_{x}$ ", the distance " $d_{x}$ " that mediates between interacting bodies" barycenters and the electro-gravitational constant " $k_{G E}$ " -is important to note that this constant in this work is related with the planet's masses and not to their constituent quarks-. This force is calculated using the following mathematical expression.

$$
F_{G E}=k_{G E} \frac{m_{S u n} m_{x}}{d_{x}^{2}},
$$

In this expression indicates the absolute inertial masses instead of planetary charges of the planetary quarks that make up -on planetary bodies due to its massive inertia mass proportionality between quarks and masses tends to a constant value, therefore we can work without problems with absolute masses-. Also, this method is a mean of simplifying the planetary calculations while maintaining the current tradition in astronomical science, which works with units of mass.

\section{Magneto-gravitational vectors}

The vector magneto-gravitational field " $\bar{B}$ " is determined by the movement of planetary quarks of the space body in rotation. It corresponds to the known Biot's Law (Jean Baptiste Biot, 1820) in the atomic field, but unlike the one used in atomic subspace fields, as shown in the Figure No. 4 , the vector field " $\bar{B}$ " that has an effect on the body interact is given by the vector field " $\overline{B_{x}}$ " that is directly the cosine of the angle " $\alpha$ " that is on the same plane of body rotation radius " $a$ ". Unlike the Biot's Law (Jean Baptiste Biot, 1820) the decrease of this force is linear and not to the square of the distance due to its spread in the planetary field is cylindrical rather than spherical spreading as in the atomic field and its representation equipotential plot points similar to the Figure No. 2 of the drag field forces. The calculation is given by the following expression.

$$
\overline{B_{x}}=k_{G M} \int \frac{\omega m_{u} a \cdot d l \times \hat{r}}{r},
$$

where " $k_{G M}$ " is the magneto-gravitational constant, " $\omega$ " is the angular velocity of rotation of the mass unit " $m_{u}$ " and " $r$ " is the line of action between the moving unit mass and any point in space. In the Figure 4 shows the direction and sense of vector field " $B$ " that belongs to the line of action " $r$ " and keeping the direction towards the rotation point on the circle of the plane " $y, x$ ". The resulting field vector " $\overline{B_{x}}$ " belonging to the axis of coordinates " $x$ ", ranges between minimum and maximum values, without changing sign, as rotating the point about " $a$ " in rotation. The result the Sun's magneto-gravitational field of the planetary subspace, determined by the vector " $\overline{B_{x}}$ ", is shaped like an ellipse of revolution with the major axis pointing to the Kuiper Belt, and its center at the central point of the Sun. The vector " $\overline{B_{y}}$ " is canceled by the covalent points of the opposite half-plane. There are no " $\overline{B_{z}}$ " transverse vectors on the plane " $y, z$ " as shown in the Figure No. 4.

\section{Magneto-gravitational force}

The magneto-gravitational force " $F_{G M}$ " that affects an object in orbit depends on whether the object is rotating on itself or not, if both objects in orbiting have rotation should be considered the rotation of both because the effects of magneto-gravitational field in addition, our Moon is a typical case because it takes a spin on its axis for Earth day, like the Earth that takes a spin on its axis for Earth day, that's why the Moon always shows the same face. The magneto-gravitational force " $F_{G M}$ " for the entire volume in the Figure No. 4 , is exclusively determined by the vector " $\overline{B_{x}}$ ".

The calculation of the magneto-gravitational force " $F_{G M}$ " Sun's interaction (subscript "o") with any planet (subscript " $"$ ") can be achieved through the following mathematical expression.

$$
F_{G M}=k_{G M} \frac{\omega_{0} r_{0} m_{0} m_{x}}{d_{x}} \operatorname{Cos}\left[\phi_{x}\right],
$$

where " $F_{G M}$ " is the magneto-gravitational force in this case determined exclusively by the Sun, " $k_{G M}$ " is the magneto-gravitational constant to planetary masses, " $\omega_{0}$ " is the Sun's angular velocity, " $r_{0}$ " is the solar equatorial radius, " $m_{0}$ " is the mass of the Sun, " $m_{x}$ " is the planet's mass interacting, " $d_{x}$ " is the distance between the barycenter of the Sun and the planet and $\phi_{x}$ is the bank angle between the straight line distance " $d_{x}$ " and the Sun's equatorial plane.

The calculation of the interaction of the planet from the Sun and the sum of both effects of magneto-gravitational 
forces can be accomplished through the following mathematical expression.

$$
\begin{aligned}
& F_{G M}=k_{G M} \frac{\omega_{x} r_{x} m_{0} m_{x}}{d_{x}} \cos \left[\theta_{x}\right] \quad \text { (Planet from the Sun) } \\
& \text { then the sum is } k_{G M} \frac{\omega_{0} r_{0} m_{0} m_{x}}{d_{x}} \cos \left[\phi_{x}\right]+k_{G M} \frac{\omega_{x} r_{x} m_{0} m_{x}}{d_{x}} \cos \left[\theta_{x}\right] \\
& \therefore \quad F_{G M}=k_{G M} \frac{m_{0} m_{x}}{d_{x}}\left(\omega_{0} r_{0} \cos \left[\phi_{x}\right]+\omega_{x} r_{x} \cos \left[\theta_{x}\right]\right),
\end{aligned}
$$

see the same references as those given for the above equations, " $\theta_{x}$ " in this case is the angle of the planet's equatorial plane to the equatorial plane of the Sun -not to be confused with the polar inclination of the planet-.

\section{Resulting inertial objects in orbit}

The resulting inertial of tangential escape " $F$ " of objects in orbit is established through the following expression.

$$
F_{I}=\frac{m_{x} v_{x}^{2}}{r_{x}}=m_{x} \omega_{x}^{2} r_{x},
$$

where " $m_{x}$ " is the object's mass in orbit, " $v_{x}$ " is the average tangential velocity at the point of measurement, " $r_{x}$ " is the radius of curvature of the virtual ellipse that is the planet orbit at the point of measurement, and " $\omega_{x}$ " is the speed instantaneous angular object in orbit at the point of measurement.

\section{Planetary stability}

An object (planet, moon, artificial satellite or comet, etc.) remains in orbit only if at the instant of crossing over the main equatorial plane (points of the aphelion and perihelion) the sum of all interacting forces tends to zero. In any other point of the orbit there are two unbalanced vectors that determine the one hand (vector sum of " $\overline{R_{1}}$ " and " $\overline{R_{2}}$ " on the line "a") the acceleration -positive (from the aphelion to the perihelion) or negative (from the perihelion to aphelion)- and on the other (vector " $\overline{R_{3}}$ ”) the advance or pitch of the elliptical helix. In Figure No. 5 shows a diagram of action of gravitational forces interact on the point "P" between the Sun and any planet. These forces are: " $F_{A}$ " is the drag force of the Sun field-effect action of attraction between the Sun and the planet and with respect to the Sun's polar axis and direction given by the line " $b$ " coordinate axis parallel " $x$ " that links point " 0 " and "P", the drag plane of the solar field is parallel or belongs to the equatorial plane of the Sun -I have not mentioned for reasons of simplicity, the drag force field determined by the rotation planet adds to the solar field drag-, " $F_{G M}$ " is the magneto-gravitational force acting effect of attraction between the Sun and the planet and with direction from the planet's barycenter and radial to the Sun's barycenter, and direction given by the line "a" linking point "P" and point " 0 ", this magnetic force compared to the drag force of the solar field is very small magnitude, these three forces are the only ones with action attraction between planetary bodies, the drag force of the solar field, the drag force field planetary and the magneto-gravitational force.

The force " $F_{G E}$ " is electro-gravitational effective action of repulsion between planetary objects that interact and make sense from the barycenter of the planet and the radial barycenter to the Sun, and direction given by the line "a" linking point "P" and point " 0 ", this force exactly matches the direction of magnetic force gravitational action but its effect is opposite. Finally, there is " $F_{I}$ " which is the resultant inertial pointing outside of the orbit direction given by the radius of curvature of the virtual ellipse of the orbit at the point " $P$ " and perpendicular to its tangent and the tangent plane belonging to the helix through the barycenter of the Sun or central body.

As the result of these forces have two options.

Alternative A: If the barycenter of the planet -point "P"- does not belong to the Sun's equatorial plane, the sums of all action effects determine three resultants. Vector " $\overline{R_{1}}$ ", is the resultant vector acting effect of attraction because it is the sum of the forces acting effect of attraction -the solar field drag, dragging planetary field, magneto-gravitational force of the Sun and magneto-gravitational force on the planet, all with action attraction effect-. We can see that the direction of the resultant " $\overline{R_{1}}$ " does not point to the Sun's barycenter, but at an intermediate point on the polar axis of the Sun, which is between the Sun barycenter and point " $0_{x}$ " plane of action of solar field drag. Vector " $\overline{R_{2}}$ ", is the resultant vector acting effect of repulsion between the Sun and the planet, because it is the sum of the forces acting effect opposite to attraction -the electro-gravitational force of the Sun and the planet and tangential force of inertia resulting from the planet-. Vector " $\frac{R_{3}}{}$ ", is the vector resulting from the vector sum of " $\overline{R_{1}}$ " and vector " $\overline{R_{2}}$ " because we must take into account the dihedral angle 
formed by planes belonging to these vectors, this is because the two vectors -“ $\overline{R_{1}}$ " and " $\overline{R_{2}}$ "- are at different planes of action. Vector " $\overline{R_{3}}$ " determines the progress of the virtual plane of the ellipse and in terms of size step provides for the extension of the ellipse of revolution that characterized the entire planetary orbit. In this alternative the sum of the vectors " $\overline{R_{1}}$ " and " $\overline{R_{2}}$ " on the line "a" is always different from zero - greater than zero when the planet is on the ellipse in the direction towards the Sun, from the aphelion to the perihelion, increasing its speed due to the positive acceleration, and less than zero when the planet is on the ellipse from the Sun, from the perihelion to aphelion, slowing down due to negative acceleration-.

Alternative B: If the planet's barycenter -point "P"- belongs to the Sun equatorial plane, the sums of all action effects determine only two resultants. This occurs in only two points of any planetary orbit, the perihelion and aphelion which corresponds to the moment of crossing the equatorial plane of the Sun In this case we have only the vector " $\overline{R_{1}}$ " and vector " $\overline{R_{2}}$ " -both vectors are necessarily equal and belong to the Sun's equatorial planeconsequently there is no resultant vector " $\overline{R_{3}}$ ". In all subsequent calculations we will use this option because only in just these two points of any orbit is satisfied that the equilibrium equation gives the vector resulting " $\overline{R_{3}}$ " equal to zero and therefore these two points in the acceleration of the body in orbit tends to zero, a situation that greatly simplifies the calculations.

The principle of equilibrium of any objects in orbit and set out in perihelion and aphelion to comply with the B alternative can be formulated with the following mathematical expression.

$$
\widetilde{F_{A x}}+\widetilde{F_{G M x}}-\overrightarrow{F_{I x}}-\overline{F_{G E x}}=0,
$$

where " $F_{A x}$ " is the vector due to the drag force field of the Sun on the planet " ", " $F_{G M x}$ " is the vector due to magneto-gravitational force of Sun planetary subspace and the planet " $x$, " $F_{I x}$ " is the vector due the resulting inertial of the planet and " $F_{G E x}$ " is the vector due to the electro-gravitational force of the global subspace between the Sun and the planet " ".

\section{Determination of planetary constants}

From the equilibrium equation ultimately view (13) and applying equations (6), (11), (12) and (7) respectively, we can calculate the magnitudes of the planetary constants. With this objective from the currently known data of Mercury and Earth, these two planets are chosen, because one has the highest closeness to the Sun -the forces to which it is subjected are the top- and Earth, because it is the planet that we know more precisely the mass and the parameters of aphelion and perihelion.

The equilibrium equation to solve in aphelion or perihelion is the following.

$$
\begin{aligned}
& \widetilde{F_{A x}}+\widetilde{F_{G M x}}-\overrightarrow{F_{I x}}-\overrightarrow{F_{G E x}}=0 \\
& \quad \therefore \quad Y_{P} \rho_{0} \omega_{0} \frac{\pi^{2} r_{0}^{2} r_{x}^{3}}{3 d_{x}^{2}}+\frac{\left|k_{G E}\right|}{\left|c^{4}\right|} \frac{m_{0} m_{x}}{d_{x}}\left(\omega_{0} r_{0}+\omega_{x} r_{x}\right)-m_{x} \psi_{x}^{2} d_{x}-k_{G E} \frac{m_{0} m_{x}}{d_{x}^{2}}=0,
\end{aligned}
$$

where the subscripts indicate the variables: " 0 " is a variable belonging to the Sun and " $x$ " is a variable belonging to a planet in our solar system.

The first equation of the pair of equations used in the final resolution is as follows. See the Appendix.

$$
Y_{P} \rho_{0} \omega_{0} \frac{\pi^{2} r_{0}^{2} r_{1}^{3}}{3 d_{m 1}^{2}}+\frac{\mid k_{G E}}{\left|c^{4}\right|} \frac{m_{0} m_{1}}{d_{m 1}}\left(\omega_{0} r_{0}+\omega_{1} r_{1}\right)-m_{1} \psi_{m 1}^{2} d_{m 1}-k_{G E} \frac{m_{0} m_{1}}{d_{m 1}^{2}}=0,
$$

where the subscripts of the variables indicate the following: “" $"$ is for the minimum distance to the Sun reached at perihelion, " 0 " is a variable Sun's characteristic, " $l$ " is a variable characteristic of Mercury to be the first planet of the solar system, with the addition of this subscript, and the variables are detailed in the preceding paragraphs. In this equation I replaced the magneto-gravitational constant " $k_{G M}$ " by the ratio between the absolute values the electro-gravitational constant " $k_{G E}$ " and the fourth power of the speed of light, see the Einstein field equations EFE (Einstein Albert, 1915), thus we have only two unknowns to solve the pair of equations, " $Y_{P}$ " the Sun's drag constant of the subspace planetary and " $k_{G E}$ " electro-gravitational constant also from subspace planetary.

The second equation used in the pair of equations is as follows. In this equation as can be seen only have replaced the variables of the perihelion of Mercury by the variables currently estimated for the Earth aphelion, the subscript " $m$ " was changed from lowercase to " $M$ " capitalized and the subscript " $l$ " is replaced by the 
subscript " " identifies the Earth.

$$
Y_{P} \rho_{0} \omega_{0} \frac{\pi^{2} r_{0}^{2} r_{3}^{3}}{3 d_{M 3}^{2}}+\frac{\mid k_{G E}}{\left|c^{4}\right|} \frac{m_{0} m_{3}}{d_{M 3}}\left(\omega_{0} r_{0}+\omega_{3} r_{3}\right)-m_{3} \psi_{M 3}^{2} d_{M 3}-k_{G E} \frac{m_{0} m_{3}}{d_{M 3}^{2}}=0,
$$

where " $M$ " is the maximum distance reached the Sun at aphelion. The expression obtained for the constant electro-gravitational of planetary subspace " $k_{G E}$ ", is the follow.

$$
k_{G E} \rightarrow \frac{\left|c^{4}\right|\left(d_{m 1}^{3} m_{1} r_{3}^{3} \psi_{m 1}^{2}-d_{M 3}^{3} m_{3} r_{1}^{3} \psi_{M 3}^{2}\right)}{m_{0}\left(m_{1} r_{3}^{3}\left(d_{m 1}\left(r_{0} \omega_{0}+r_{1} \omega_{1}\right)-\left|c^{4}\right|\right)+m_{3} r_{1}^{3}\left(\left|c^{4}\right|-d_{M 3}\left(r_{0} \omega_{0}+r_{3} \omega_{3}\right)\right)\right)}
$$

The expression obtained for the Sun's constant drag of planetary subspace $Y_{P}$, is the follow.

$$
\begin{aligned}
Y_{P} \rightarrow 3 m_{1} m_{3}\left\{d_{m 1} d_{M 3}^{3} \psi_{M 3}^{2}\left(r_{0} \omega_{0}+r_{1} \omega_{1}\right)+d_{m 1}^{3} \psi_{m 1}^{2}\left(r_{0} \omega_{0}+r_{3} \omega_{3}\right)-\left|c^{4}\right| d_{M 3}^{3} \psi_{M 3}^{2}\right\} & \\
& \left\{\left(\pi^{2} r_{0}^{2} \rho_{0} \omega_{0}\right)\left(m_{1} r_{3}^{3}\left\langle d_{m 1}\left(r_{0} \omega_{0}+r_{1} \omega_{1}\right)-\left|c^{4}\right|\right\rangle+m_{3} r_{1}^{3}\left\langle\left|c^{4}\right|-d_{M 3}\left(r_{0} \omega_{0}+r_{3} \omega_{3}\right)\right\rangle\right)\right\}^{-1}
\end{aligned}
$$

Using data from the Appendix and taking only the magnitudes of the radius and mean velocities of the orbits of Mercury, Earth and planetary constants were calculated with the expressions (17) and (18) -eliminating the letters " $m$ " and " $M$ " subscripts on the variables- and obtained the magnitudes shown in (19), (20) and (21). Much of the data in Appendix were obtained or calculated based on bulk parameters and orbital parameters of the Fact Sheets by NASA (NASA Goddard Space Flight Center, Greenbelt, MD 20771).

$$
\begin{gathered}
k_{G E}=2.88821919179004282377688826727 \times 10^{-10} \mathrm{~N} \cdot \mathrm{m}^{2} \cdot \mathrm{kg}^{-2} \\
k_{G M}=\frac{\left|k_{G E}\right|}{\left|c^{4}\right|}=3.57558690249434318757018569465 \times 10^{-44} \mathrm{~N} \cdot \mathrm{kg}^{-1} \\
Y_{P}=2.53265203011334180831909179688 \times 10^{9} \mathrm{~s}^{-1} \cdot \mathrm{m}^{-1}
\end{gathered}
$$

The dimensional unit $\left(\mathrm{N} \cdot \mathrm{kg}^{-1}\right)$ of the " $k_{G M}$ " magneto-gravitational constant is very different to that used in atomic electromagnetism; this is because the proportionality is referred to the masses rather than the charges of planetary quarks.

\section{Resolution of the distances to the Sun at perihelion and aphelion of the planet}

From the equilibrium equation (14) or equation (15) we can solve the expression for the calculation of the distances between barycenters of any planet at perihelion or aphelion in terms of planetary constants obtained (19), (20) and (21). Solved mathematical expressions are as follows, for the distance in perihelion is:

$$
\begin{aligned}
& d_{m x}=\left\{\begin{array}{r}
23^{1 / 3} k_{G M} m_{0} m_{x}^{2} \psi_{m x}^{2} \omega_{0} r_{0}+23^{1 / 3} k_{G M} m_{0} m_{x}^{2} \psi_{m x}^{2} \omega_{x} r_{x}+ \\
2^{1 / 3}\left(\begin{array}{r}
3 \pi^{2} m_{x}^{2} r_{0}^{2} r_{x}^{3} Y_{P} \psi_{m x}^{4} \rho_{0} \omega_{0}-9 k_{G E} m_{0} m_{x}^{3} \psi_{m x}^{4}+ \\
\sqrt{3}\left(m_{x}^{4} \psi_{m x}^{6}\left[\begin{array}{r}
3 \psi_{m x}^{2}\left(\pi^{2} r_{0}^{2} r_{x}^{3} Y_{P} \rho_{0} \omega_{0}-3 k_{G E} m_{0} m_{x}\right)^{2}- \\
4 k_{G M}^{3} m_{0}^{3} m_{x}^{2}\left(r_{0} \omega_{0}+r_{x} \omega_{x}\right)^{3}
\end{array}\right)^{1 / 2}\right.
\end{array}\right)^{1 / 3}
\end{array}\right\} .
\end{aligned}
$$

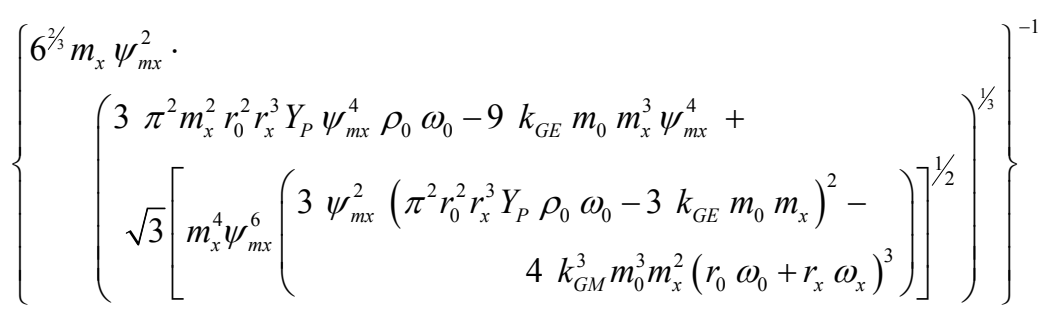


for the distance in aphelion is:

$$
\begin{aligned}
& d_{M x}=\left\{\begin{array}{l}
23^{1 / 3} k_{G M} m_{0} m_{x}^{2} \psi_{M x}^{2} \omega_{0} r_{0}+23^{1 / 3} k_{G M} m_{0} m_{x}^{2} \psi_{M x}^{2} \omega_{x} r_{x}+ \\
2^{1 / 3}\left[\begin{array}{l}
3 \pi^{2} m_{x}^{2} r_{0}^{2} r_{x}^{3} Y_{P} \psi_{M x}^{4} \rho_{0} \omega_{0}-9 k_{G E} m_{0} m_{x}^{3} \psi_{M x}^{4}+ \\
\sqrt{3}\left[m_{x}^{4} \psi_{M x}^{6}\left(\begin{array}{r}
3 \psi_{M x}^{2}\left(\pi^{2} r_{0}^{2} r_{x}^{3} Y_{P} \rho_{0} \omega_{0}-3 k_{G E} m_{0} m_{x}\right)^{2}- \\
\left.4 k_{G M}^{3} m_{0}^{3} m_{x}^{2}\left(r_{0} \omega_{0}+r_{x} \omega_{x}\right)^{3}\right)
\end{array}\right)^{1 / 2}\right.
\end{array}\right\} .
\end{array}\right. \\
& \left\{\begin{array}{l}
6^{2 / 3} m_{x} \psi_{M x}^{2} \cdot \\
\left.\sqrt{3}\left[\begin{array}{l}
\left.3 \pi^{2} m_{x}^{2} r_{0}^{2} r_{x}^{3} Y_{P} \psi_{M x}^{4} \rho_{0} \omega_{0}-9 k_{G E} m_{0} m_{x}^{3} \psi_{M x}^{4}+\pi^{2} r_{0}^{2} r_{x}^{3} Y_{P} \rho_{0} \omega_{0}-3 k_{G E} m_{0} m_{x}\right)^{2}- \\
\left.4 k_{G M}^{3} m_{0}^{3} m_{x}^{2}\left(r_{0} \omega_{0}+r_{x} \omega_{x}\right)^{3}\right)
\end{array}\right]^{1 / 2}\right)^{1 / 3}
\end{array}\right\}
\end{aligned}
$$

\section{Solving the equation for calculating the solar system planetary masses}

Also from the equations of equilibrium (15) or equation (16) and by replacing the subscript " 1 " or subscript " 3 " by the subscript " $x$ " to identify any planet and the constants $k_{G E} / \mathrm{c}^{4}$ by the constant $k_{G M}$; we solve the expression for calculating the magnitude of the planetary masses as a function of planetary constants. Solved mathematical expressions are as follows, based on data of perihelion (subscript “ ${ }^{m}$ ") is:

$$
m_{x}=\frac{\pi^{2} r_{0}^{2} r_{x}^{3} Y_{P} \rho_{0} \omega_{0}}{3\left(d_{m x}^{3} \psi_{m x}^{2}+m_{0}\left(k_{G E}-d_{m x} k_{G M}\left(r_{0} \omega_{0}+r_{x} \omega_{x}\right)\right)\right)}
$$

Based on data from the aphelion (subscript “ $M$ ”) is:

$$
m_{x}=\frac{\pi^{2} r_{0}^{2} r_{x}^{3} Y_{P} \rho_{0} \omega_{0}}{3\left(d_{M x}^{3} \psi_{M x}^{2}+m_{0}\left(k_{G E}-d_{M x} k_{G M}\left(r_{0} \omega_{0}+r_{x} \omega_{x}\right)\right)\right)},
$$

where the subscripts " " should be replaced by the number of the planet we want to calculate the corresponding mass.

\section{Solar system planetary masses}

The results in the calculation of the masses of all known planets in our solar system in terms of the expression (24) in Tables No. 1 and the expression (25) in Tables No. 2; are shown using the known quantities today. In this case the calculations were made considering the sum of the planet's mass estimated today most of its satellites (see column "Current mass"), using the constants (19), (20) and (21), also had to recalculate the corresponding virtual radius of the planets, taking into account the currently estimated densities, the calculation is performed with the expression (26) and $m_{x}$ with mass of the planet more its satellites.

$$
r_{x}=\sqrt[3]{\frac{3 m_{x}}{4 \pi \rho_{x}}},
$$

The density figures included in the tables below with the title "Calc. density" are calculated using the expression (27). The densities on all tables are in kg. $\mathrm{m}^{-3}$.

$$
\rho_{x}=\frac{3 m_{x}}{4 \pi r_{x}^{3}},
$$

The following Table No. 3 and Table No. 4 the same way as in the previous tables were re-perform the same calculations but taking the masses of the planets without considering the mass of its satellites.

In the Tables No. 1, 2, 3 and 4, significant differences were found for each planet mass as a function of perihelion and aphelion. This anomaly is due to errors in the quantities used in the calculations, masses and distances from the Sun and/or speeds.

\section{Verification Earth's orbital data}

Shows the distances calculated in the Table No. 5 for the perihelion and aphelion, the mass and estimated 
differences from today to the Earth. Also consider that the magnitude of mass calculated and the estimation today exactly matches because the Earth it is the reference point, taken as correct in this work.

\section{Verification Mercury's orbit data}

Shows the distances calculated in the Table No. 6 for the perihelion and aphelion, the mass and differences from those estimated for Mercury now proceeding in the same way as for the Earth and on the assumption that the mass of Mercury was correct.

In the Table No. 7 is again calculated the average mass of Mercury but with the assumption that the distances of perihelion and aphelion are correct. Then with the average mass is recalculated and aphelion distances of perihelion and with these new units are controlled new mass. I believe that in the case of Mercury we have an underestimation of its mass and small differences in the calculation of the magnitudes of astronomical distances perihelion and aphelion.

\section{Planetary velocity recalculated}

In order to have a clear vision of what happens to the differences in planetary magnitudes; were recalculated velocities at aphelion and perihelion points. The calculation was performed using the following expression obtained starting the expression (24) or (25) and by eliminating the " $\mathrm{m}$ " and " $\mathrm{M}$ " subscripts.

$$
\psi=\sqrt{Y_{P} \rho_{0} \omega_{0} \frac{\pi^{2} r_{0}^{2} r_{x}^{3}}{3 m_{x} d_{x}^{3}}+k_{G M} \frac{m_{0}}{d_{x}^{2}}\left(\omega_{0} r_{0}+\omega_{x} r_{x}\right)-k_{G E} \frac{m_{0}}{d_{x}^{3}}},
$$

The magnitude of the velocity on the perihelion calculated for each planet can be seen in Table No. 8 .

Based on the calculated velocities in the first place and by means of expressions (24) and (25) we calculated the masses of all planets, and then using expressions (22) and (23) distances were calculated; the distances in perihelion and aphelion coincided exactly with the values currently estimated, therefore not shown the values obtained for this reason.

\section{Recalculation of planetary masses and distances}

To correct and consistent results in all the planets in our solar system, we recalculated the distances of each planet with the new average mass and function were recalculated including the respective planetary masses. The magnitude of the perihelion calculated for each planet can be seen in the Table No. 9, and the magnitude of the aphelion calculated for each planet can be seen in the Table No. 10.

With the magnitude of the perihelion and aphelion in Tables No. 9 and 10 we recalculated the masses of all the planets (seen in the Table No. 11). I should clarify that in this case exactly matches the magnitude of the calculated mass for each planet, both for the aphelion to the perihelion.

Regarding the current estimate, it is remarkable the difference in the calculated mass planets whose distance from the Sun are larger than the Earth, also highlights the similarity of the densities of all the planets, very different to the current concept that gives masses densities very close to the water from Jupiter to Pluto.

\section{Calculations of planetary satellites}

In calculating the dimensions concerning planetary satellites we must take into account an important Solar phenomenon, the Sun trailing the field in inverse proportion to the distance, this phenomenon implies that the level of the Sun's surface to stretch the field is maximum and decreases as a function of distance to consider. Due to the small mass of the planets respect to the Sun's mass; at distances close to the Sun, this massive pre-stressed of the planetary field due to the Sun, determines that planets such as Mercury or Venus, can only produce minimal drag planetary field around them, because the planetary field is already enormously strained by the Sun, while the planets that are far away from the Sun can produce a much greater drag of the planetary field, because the planetary field at these distances is in much less strained. This phenomenon of stretching the field closest to the Sun determines the absence of natural satellites of the planets Mercury and Venus, because orbits are possible only at very low or near the surface and also very low speeds, while at greater distances from the Sun the amount satellite increases markedly, especially in the case of Jupiter due to its distance from the Sun and its enormous mass, the amount is very large of natural satellite. To enter this analysis we must first understand that is the gravitational constant; something we see in the next section.

\section{Gravitational constant}

Starting from the global equilibrium equation (14); by replacing the constants kGE/c4 by the constant kGM and incorporating the drag coefficient field correction " $\mathrm{x}$ ", proper to each planet due the density of the planetary 
field that surrounds, that is in inverse proportion to the distance you have respect to the Sun's barycenter, be established the equilibrium equation of the satellites as we see in expression (29).

$$
Y_{P} \varsigma_{x} \rho_{x} \omega_{x} \frac{\pi^{2} r_{x}^{2} r_{y}^{3}}{3 d_{y}^{2}}+k_{G M} \frac{m_{x} m_{y}}{d_{y}}\left(\omega_{x} r_{x}+\omega_{y} r_{y}\right)=m_{y} \psi_{y}^{2} d_{y}+k_{G E} \frac{m_{x} m_{y}}{d_{y}^{2}},
$$

where the subscripts " $x$ " (the Sun's data previously) identifies the planet's own data, while the subscript " $y$ " (the planet's data previously) identifies the satellite data, see Appendix.

From the equilibrium equation (29) solve for the drag coefficient field correction " $c \mathrm{x}$ " and we obtain the expression (30).

$$
\varsigma_{x}=\frac{3 m_{y}\left\{d_{y}^{3} \psi_{y}^{2}+m_{x}\left[k_{G E}-d_{y} k_{G M}\left(\omega_{x} r_{x}+\omega_{y} r_{y}\right)\right]\right\}}{\pi^{2} r_{x}^{2} r_{y}^{3} Y_{P} \rho_{x} \omega_{x}}
$$

We verify the calculations developed by replacing the coefficient YP, by the second member in expression (30), in the first member of expression (29) and then simplify this member.

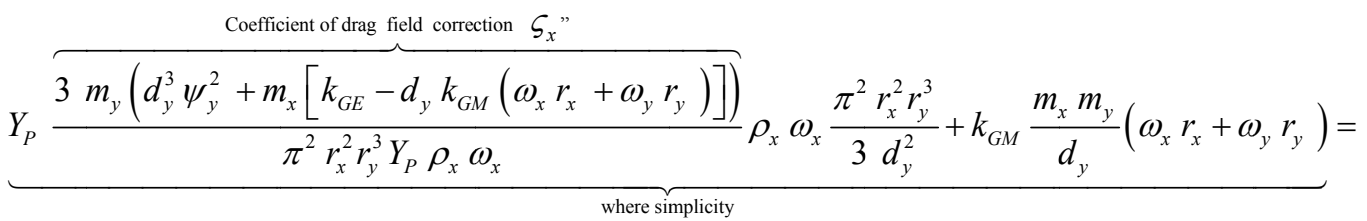

$$
\begin{aligned}
& m_{y} \psi_{y}^{2} d_{y}+k_{G E} \frac{m_{x} m_{y}}{d_{y}^{2}} \\
& m_{y} \psi_{y}^{2} d_{y}+k_{G E} \frac{m_{x} m_{y}}{d_{y}^{2}}=m_{y} \psi_{y}^{2} d_{y}+k_{G E} \frac{m_{x} m_{y}}{d_{y}^{2}}
\end{aligned}
$$

After we verifying the expression, we make a transformation as follows: In the first place we spent the electro-gravitational repulsive force of the second member to the first member of the expression and in the second place, simplify only the first and second terms of the first member (the terms the drag field and electro-gravitational); after reordering all the first member, we extract the common factors, as shown in the steps of the expression (32).

$$
\begin{gathered}
\underbrace{\frac{3 m_{y}\left(d_{y}^{3} \psi_{y}^{2}+m_{x}\left(k_{G E}-d_{y} k_{G M}\left(\omega_{x} r_{x}+\omega_{y} r_{y}\right)\right)\right)}{\pi^{2} r_{x}^{2} r_{y}^{3} Y_{P} \rho_{x} \omega_{x}} \rho_{x} \omega_{x} \frac{\pi^{2} r_{x}^{2} r_{y}^{3}}{3 d_{y}^{2}}-k_{G E} \frac{m_{x} m_{y}}{d_{y}^{2}}}_{P}+ \\
k_{G M} \frac{m_{x} m_{y}}{d_{y}}\left(\omega_{x} r_{x}+\omega_{y} r_{y}\right)=m_{y} \psi_{y}^{2} d_{y} \\
\text { then } \quad \frac{d_{y}^{2} \psi_{y}^{2} m_{y}}{d_{y}}-k_{G A} \frac{m_{x} m_{y}}{d_{y}}\left(\omega_{x} r_{x}+\omega_{y} r_{y}\right)+k_{G M} \frac{m_{x} m_{y}}{d_{y}}\left(\omega_{x} r_{x}+\omega_{y} r_{y}\right)= \\
\text { separate the common factor } \quad \frac{d_{y}^{2} \psi_{y}^{2}}{m_{x}} \frac{m_{x} m_{y}}{d_{y}}=m_{y} \psi_{y}^{2} d_{y}
\end{gathered}
$$

Finally, multiply both sides of the expression (32) per unit depending on the magnitude of the distance, simplifying and rearranging we obtain the mathematical expression of the gravitational constant.

$$
\begin{gathered}
\text { being the unit } 1=\frac{d_{y}}{d_{y}} \text { then } \frac{d_{y}}{d_{y}} \frac{d_{y}^{2} \psi_{y}^{2}}{m_{x}} \frac{m_{x} m_{y}}{d_{y}}=\frac{\chi_{x}}{d_{y}} m_{y} \psi_{y}^{2} d_{y} \\
\text { and rearranging it } \underbrace{\frac{d_{y}^{3} \psi_{y}^{2}}{m_{x}}}_{\downarrow} \frac{m_{x} m_{y}}{d_{y}^{2}}=m_{y} \psi_{y}^{2} d_{y} \\
\text { Gravitational constant (G) and Newton equation }
\end{gathered}
$$

Note that the gravitational constant (33) determined by the variables involved in its expression is specific to each planet and consequently it cannot consider the gravitational constant as a universal constant. Newton's equation 
(Newton Sir Isaac, 1686) is only valid to some extent and with limitations, for the calculation of the planetary satellites, only in the case to know previously and the precise value of the gravitational constant characteristic of each the planet. Still another very important aspect to consider is, if we use the Newton's expression (33) to calculate the coupling strength of the satellites, the results will be magnitudes of coupling forces in orbit much lower than reality, because in this expression does not include the electro-gravitational repulsive force determined by the constant "kGE" that in all cases must be added to the resultant inertial of the satellites in orbit. In the case of planetary calculations used in the Newton's expression (33), as has been done so far, due that by ignoring the electro-gravitational repulsive force, the planets that are farther from the Sun appear to be less dense than the real.

Using the calculations of the gravitational constant and the resultant repulsive action for three known planetary satellites for many years, and plus the very recently discovered satellite of Pluto, Charon, we obtain the results shown in Table No. 12. In the case of the gravitational constant "G" is a averaged with the current data of the perihelion and aphelion, in the calculations we used the following expressions.

$$
\begin{gathered}
G=\frac{1}{2}\left(\frac{d_{m y}^{3} \psi_{m y}^{2}}{m_{x}^{2}}+\frac{d_{M y}^{3} \psi_{M y}^{2}}{m_{x}}\right) \\
R_{R-\text { Newton }}=m_{y} \psi_{y}^{2} d_{y} \\
R_{R-T C S}=m_{y} \psi_{y}^{2} d_{y}+k_{G E} \frac{m_{x} m_{y}}{d_{y}^{2}}
\end{gathered}
$$

The calculations were made with the current estimate of the magnitudes of mass and distance. The estimated magnitude of the gravitational constant for Earth is within the limits -over seven hundred thousandths- of the present Newtonian constant of gravitation with a magnitude of $6.67428(67) \times 10-11 \mathrm{~m} 3 \mathrm{~kg}-1 \mathrm{~s}-2$ published by the National Institute of Standards and Technology (NIST) (Mohr Peter J., et al. 2007). In the case of Pluto's satellite Charon, it is clear that there is an overestimation of the at perihelion and aphelion distances and/or the average speed on these two points in the solar equatorial plane, or the mass of Pluto is very understated -which is most likely-, see Table No. 11.

\section{The gravity acceleration}

From the expression (33) we can make theoretical calculations of the acceleration of gravity at the surface of the Earth and Moon, because it is subtracted on the equilibrium expression (16) the magnitude of electro-gravitational force. To calculate the acceleration of gravity at the Earth's surface, taking the first member of expression (33) and if the mass and the distances are carried to the dimensional limits we get the expression (37).

$$
\overbrace{\left(\frac{d_{y}^{3} \psi_{y}^{2}}{m_{x}}\right)}^{\text {Constant "G" }} \cdot \lim _{\substack{m_{y} \rightarrow 1 \\ d_{y} \rightarrow r_{x}}}\left[\frac{m_{x} m_{y}}{d_{y}^{2}}\right]=\frac{d_{y}^{3} \psi_{y}^{2}}{r_{x}^{2}} \quad \text { (37), calculating by the Earth: } G_{3}=\frac{d_{M}^{3} \psi_{M}^{2}}{r_{3}^{2}}=9.831076472 \mathrm{~m} . \mathrm{s}^{-2}
$$

The observed difference in acceleration is due to the Moon's angular velocity average, we can calculate this velocity by solving the following equation.

$$
\text { being } \frac{d_{M}^{3} \psi_{M}^{2}}{r_{3}^{2}}=g=9.80665 \mathrm{~m} . \mathrm{s}^{-2} \text { (current), then } \psi_{M}=\frac{r_{3} \sqrt{g}}{d_{M}^{3 / 2}}=2.65019 \times 10^{-6} \text { radians. } \mathrm{s}^{-1}
$$

The Moon's angular velocity theoretical calculated is $1 \%$ lower than the estimated magnitude nowadays, of $2.65349 \times 10-6$ radians.s- 1 . To avoid differences also calculated the theoretical mass of the Moon based on the angular velocity that we have calculated, we solve from the expression (33).

being $\overbrace{\left(\frac{d_{M}^{3} \psi_{M}^{2}}{m_{3}}\right)}^{\text {Constant "G" }} \cdot \frac{m_{M}}{r_{M}^{2}}=g_{\text {Moon }}=1.622 \mathrm{~m} \cdot \mathrm{s}^{-2}$ (current),

$$
\text { then } m_{M}=\frac{g_{\text {Moon }} m_{3} r_{M}^{2}}{d_{M}^{3} \psi_{M}^{2}}=7.518190971 \times 10^{22} \mathrm{~kg}
$$

Consider that theoretical mass of Moon is $2 \%$ higher than the estimated nowadays, of $7.349 \times 10^{22} \mathrm{~kg}$.

Finally, we make the calculations of surface acceleration at the Earth and the Moon on the basis of new 
theoretical angular velocity and mass of the Moon.

$$
\text { Earth surface acceleration } \frac{d_{M}^{3} \psi_{M}^{2}}{r_{3}^{2}}=\frac{\left(3.844 \times 10^{8}\right)^{3} \cdot\left(2.65019 \times 10^{-6}\right)^{2}}{\left(6.3781 \times 10^{6}\right)^{2}}=9.80665 \mathrm{~m} \cdot \mathrm{s}^{-2} \text { (exact) }
$$

Moon surface acceleration

$$
\frac{d_{M}^{3} \psi_{M}^{2}}{m_{3}} \cdot \frac{m_{M}}{r_{M}^{2}}=\frac{\left(3.844 \times 10^{8}\right)^{3} \cdot\left(2.65019 \times 10^{-6}\right)^{2} \cdot 7.518190971 \times 10^{22}}{5.9736 \times 10^{24} \cdot\left(1.7381 \times 10^{6}\right)^{2}}=1.662 \mathrm{~m} \cdot \mathrm{s}^{-2}(\text { exact })
$$

\section{Recalculating Moon's data}

We performed the following data calculation of the Moon. The drag coefficient field correction " $\varsigma_{3}$ " is $2.2313002857048979 \times 10^{-4}$ (dimensionless) for Earth, data obtained with the expression (30). With introducing of this coefficient in the indicated expressions of the table No 13 was obtained the data shown.

\section{The Kuiper belt}

The Kuiper Belt occupies an area of the Sun's equatorial plane where the solar drag effect is minimal and reaches the area of the equatorial plane where the solar drag effect vanishes completely, because in this area, the magnitude planetary field entrainment for a given body is equal to the magnitude of the electro-gravitational repulsive force.

\section{Planetary field, the Hills cloud and the Oort cloud}

The planetary field consists mainly of solar drag lenticular shaped or ellipse of revolution, whose midplane is the Sun's equatorial plane and its axis is the Sun's polar axis to be located at the geometric center of the ellipse of revolution. Within the ellipse of revolution forces are determined primarily attractive object and toward the Sun, the maximum intensity of the forces are on the equatorial plane of the Sun or even mid-plane of the ellipse of revolution and are decreasing in a cross this plan as they approach the periphery of the ellipse of revolution. Outside this ellipse of revolution of the scope and appeal outside a null action, the forces interact on any item are repulsive, due to electro-gravitational phenomenon of quarks that make up the Sun and all matter. Between the inner zone of action outside the area attractive and repulsive action, there is a neutral zone where no action is manifest effects of attraction or repulsion and also where is located the Hills cloud or first layer of the Oort cloud, laden with a huge number of asteroids in constant random motion due to wave phenomenon of the Sun that causes collisions between the bodies in it. These conflicts in certain circumstances make some bodies of the Hills cloud to enter into the area of solar attraction and penetrate the global area of our system by adopting the characteristics of a comet.

The reason for this remarkable zonal separation of forces of action of the Sun, is due primarily to the phenomenon of solar drag has the form of infinite planes parallel to the Sun's equatorial plane, with a decrease in the intensity of action towards the solar poles also in terms of distance, while the opposite phenomenon of electro-gravitational action is always radial direction between the barycenter of the interacting objects and the Sun. These two phenomena together are the reason why the field of solar drag attraction has a lenticular cloud; the Oort cloud integrates all the action area solar zero, while the Hills cloud is located in the inner boundary of no action area.

\section{Summary}

Undoubtedly, the planetary drag field is the phenomenon that determines the stability of planetary and satellite orbits, recall that A. Einstein in the case study of the anomalies in Mercury made an approach to this work, but conceptually a different way because the deformation of the field attributed it to just the mass of the Sun or Earth, whereas in the present work attributed to the movement of planetary quarks trailing behind the planetary field by the rotation of the Sun or planet to a limit in which and can flow or slip into the planetary field, keeping tension on the surrounding environment. Very different from what happens to the atomic field of the Earth or any planet or star-known as the Earth magnetic field-in which there is a breaking of symmetry atomic subspace of atomic field surrounding in the area known as the exosphere, producing permanent drag atomic field that integrates the Earth along the entire path of the orbit. This drag is due to the enormous charges of atomic quarks, while in contrast the much smaller planetary quarks can only produce the load slip and drag the planetary field proper of planetary subspace. The drag of the atomic field in the atomic subspace, determine between the atoms of matter and between molecules the forces that keep them close together -called long as Van der Waals forces-.

The new concept of planetary drag field with the phenomenon of electro-gravitational repulsion first published in 
this paper have huge implications in astronomy, because they explain in a coherent way through the equilibrium expression (14) why two stars may be very close together and even exchange material between them without going to collapse. A bad example is worth to have a clear idea: "is like the Italians can attach the spaghettis at the fork". Also we now have a coherent explanation of globular clusters with millions of stars -why not call them planetary systems?- That move randomly within a virtual huge area without this comes to collapse, as would be expected if there was only an attractive force between the various celestial bodies.

If only there was a sense of gravitational attraction between celestial bodies, all would be chaos crash, could not exist in globular clusters, because all stars eventually collapse and collapse all galaxies and also became one mass of matter collapsed. In galaxies would have thousands of collisions of stars per day or per minute and everything would be lit by constant nova. That does not happen, why? For all the planetary systems (all stars) when they approach each other they repel each other due to the electro-gravitational interaction repulsive action. Thus planetary systems in galaxies or in globular clusters behave the same way, as atoms in a gas -Brownian motion-, without ever colliding with each other, are closer than its individual components.

But there are other galactic level phenomena caused by interactions of the quarks that make up the subspace galactic field, this is another level of interactions that determines sense of attraction forces that cause all the planetary systems (stars) within a galaxy or globular cluster remain close together. This appeal from the galactic quarks is equal to the force of attraction of the atoms at long distances over the next atoms that surround it, remarkably similar to what happens in any substance, while the electro-gravitational interaction prevents that planetary systems (stars) collide with each other, which is equivalent to the atomic level with the electrostatic forces of the peripheral electrons avoid collisions with other atoms nearby.

My intention in this work was in the possible make calculations of the constants and planetary masses as accurately as possible, which can be seen in the tables and views, but other than the Sun, Earth and Moon, all the other planets and its satellites is seen that the magnitudes of distances in the perihelion and aphelion and speeds at these points were modified on the actual quantities measured or observed -see Tables 1 to 4 that endorse this remark-. I believe they've made these changes in the magnitudes observed in a healthy effort to bring them to acceptable levels within the only currently viable theory, the theory published in 1685 by Sir Isaac Newton (Newton Sir Isaac, 1686) which has already served more than three centuries and as we see in expression (33) despite its limitations still maintains full force.

Due to the alteration of the observed data, published data do not conform to the true values astronomical data as much as I looked, unfortunately I could not find within the vast amount of other supporting data.

It is highly desirable in the future be issued in all cases the magnitudes of perihelion and aphelion -distance and speed- of all the planets with respect to the barycenters of the Sun and the planet in the solar equatorial plane and in the case of satellites over the barycenters of the planet and the satellite in the equatorial plane of the planet, not the plane of the ecliptic as it does so far, in this way avoids a complex angular transformation prior to any calculation. Also, should be included the resulting uncertainty in terms of instruments and measurement method used and of course, using the standard dimensions of SI.

A very important aspect that emerges from this study to consider in the planetary and interplanetary navigation with current technological resources and significantly reduces the traveling time and fuel consumption is as follows:

When the path of travel is in a direction away from the Sun, the vehicle must be launched along the polar axis of planet of origin and must describe a trajectory belonging to a transverse plane with respect to the equatorial plane of the Sun, because in this direction reduces the effect of solar drag and increases the electro-gravitational repulsive effect.

In all cases of launches to the outside of our solar system the direction should also be transverse to the equatorial plane of the Sun and if you want the maximum possible acceleration the vehicle must be launched in a direction that belongs to the Sun's polar axis where the attractive forces are due to solar minimum drag and electro-gravitational repulsive forces are maximum.

In contrast, whenever the vehicle's path is heading towards the Sun, the trajectory must belong to the equatorial plane of the Sun to obtain the maximum momentum due to the attractive force of drag and the minimum solar electro-gravitational repulsive effect. The speeds that can be achieved in the zone of electro-gravitational repulsion depending on the type of vehicle propulsion are: If using ion thrusters it can achieve the speed of light $\left(299,792,458 . \mathrm{ms}^{-1}\right)$, while if it using thrusters the planetary field it can achieve the square of the speed of light $\left(89,875,517,873,681,764 . \mathrm{m} \cdot \mathrm{s}^{-1}\right)$, almost three hundred and twenty-three thousand trillion kilometers per hour. 


\section{Acknowledgments}

All my gratitude to Anibal Martin Caminoa Lizarralde by making comprehensible my pitiful English, this and previous works and web site, thank you very much Martin.

\section{References}

Biot Jean-Baptiste and Savart Félix, Griffiths David Jeffrey. (1999). Introduction to Electrodynamics, 3rd Edition, Prentice-Hall International Limited, London.

Caminoa Lizarralde Daniel E. (2010). The Three-dimensional Complex Space theory. Advanced Studies in Theoretical Physics, Vol. 4, no. 7, 323 - 351, Hikari Ltd.

Committee on Data for Science and Technology. (2006). CODATA Recommended Values of the Fundamental Physical Constants:2006, Mohr Peter J., Taylor Barry N., and Newell David B., NIST - National Institute of Standards and Technology, Gaithersburg, Maryland 20899-8420, December 28, USA 2007.

Coulomb Charles Augustin. (1884). Collection de mémoires relatifs à la physique, Société française de physique, available: http://books.google.com/books.

Einstein Albert. (1915). Die Feldgleichungen der Gravitation, and (1916), Die Grundlage der allgemeinen Relativitätstheorie, Berlin.

Hooke Robert. (1678). Ut tensio, sic vis, London. In The elements of mechanism, By Goodeve Thomas Minchin, available: http://books.google.com/books.

NASA Goddard Space Flight Center, Greenbelt, MD 20771. [Online] Available: http://www.nasa.gov/centers/goddard/about/fact_sheets.html

Newton Sir Isaac. (1686). Philosophiæ Naturalis Principia Mathematica.

Young Thomas. (1845). A Course of Lectures on Natural Philosophy and the Mechanical Arts, Philip Kelland, Printed for Taylor and Walton, London.

Table 1. Planetary mass calculation based on the distance of the perihelion

\begin{tabular}{|c|l|r|r|r|c|c|}
\hline No. & Planet & Calculated mass $(\mathrm{kg})$ & Current mass $(\mathrm{kg})$ & Calc./Curr. & Calc. density & Difference $\%$ \\
\hline 1 & Mercury & $3.21928457 \times 10^{23}$ & $3.302000 \times 10^{23}$ & 0.974950 & 5292.51 & $2.57-$ \\
\hline 2 & Venus & $5.09677218 \times 10^{24}$ & $4.868500 \times 10^{24}$ & 1.046890 & 5489.75 & $4.48+$ \\
\hline 3 & Earth & $6.00809076 \times 10^{24}$ & $6.047090 \times 10^{24}$ & 0.993551 & 5479.43 & $0.65-$ \\
\hline 4 & Mars & $8.81468508 \times 10^{23}$ & $6.418500 \times 10^{23}$ & 1.373320 & 5401.97 & $27.18+$ \\
\hline 5 & Jupiter & $7.79743681 \times 10^{28}$ & $1.898740 \times 10^{27}$ & 4.106640 & 5445.40 & $75.65+$ \\
\hline 6 & Saturn & $4.50204545 \times 10^{27}$ & $5.686010 \times 10^{26}$ & 7.917760 & 5439.50 & $87.37+$ \\
\hline 7 & Uranus & $3.72776088 \times 10^{26}$ & $8.684110 \times 10^{25}$ & 4.292620 & 5451.63 & $76.70+$ \\
\hline 8 & Neptune & $4.14844708 \times 10^{26}$ & $1.239220 \times 10^{26}$ & 3.347630 & 5483.42 & $98.54+$ \\
\hline 9 & Pluto & $4.24098573 \times 10^{22}$ & $1.412000 \times 10^{22}$ & 3.003530 & 5256.18 & $66.71+$ \\
\hline
\end{tabular}


Table 2. Planetary mass calculation based on the distance of the aphelion

\begin{tabular}{|c|l|r|r|r|c|c|}
\hline No. & Planet & Calculated mass $(\mathrm{kg})$ & Current mass $(\mathrm{kg})$ & Calc./Curr. & Calc. density & Difference $\%$ \\
\hline 1 & Mercury & $3.47771669 \times 10^{23}$ & $3.302000 \times 10^{23}$ & 1.053220 & 5717.37 & $5.05+$ \\
\hline 2 & Venus & $5.10955480 \times 10^{24}$ & $4.868500 \times 10^{24}$ & 1.049510 & 5503.52 & $4.72+$ \\
\hline 3 & Earth & $6.04622521 \times 10^{24}$ & $6.047090 \times 10^{24}$ & 0.999857 & 5514.21 & $0.01-$ \\
\hline 4 & Mars & $9.12988872 \times 10^{23}$ & $6.418500 \times 10^{23}$ & 1.422430 & 5595.14 & $29.70+$ \\
\hline 5 & Jupiter & $7.94228248 \times 10^{28}$ & $1.898740 \times 10^{27}$ & 4.182920 & 5546.55 & $76.09+$ \\
\hline 6 & Saturn & $4.59874659 \times 10^{27}$ & $5.686010 \times 10^{26}$ & 8.087830 & 5556.34 & $87.63+$ \\
\hline 7 & Uranus & $3.79192278 \times 10^{26}$ & $8.684110 \times 10^{25}$ & 4.366510 & 5545.46 & $77.10+$ \\
\hline 8 & Neptune & $4.16821148 \times 10^{26}$ & $1.239220 \times 10^{26}$ & 3.363580 & 5509.54 & $70.27+$ \\
\hline 9 & Pluto & $4.62097983 \times 10^{22}$ & $1.412000 \times 10^{22}$ & 3.272650 & 5727.14 & $69.44+$ \\
\hline
\end{tabular}

Table 3. Planetary mass calculation based on the distance of the perihelion

\begin{tabular}{|c|l|r|r|r|c|c|}
\hline No. & Planet & Calculated mass $(\mathrm{kg})$ & Current mass $(\mathrm{kg})$ & Calc./Curr. & Calc. density & Difference \% \\
\hline 1 & Mercury & $3.21928457 \times 10^{23}$ & $3.3020 \times 10^{23}$ & 0.974950 & 5292.51 & $2.57-$ \\
\hline 2 & Venus & $5.09677218 \times 10^{24}$ & $4.8685 \times 10^{24}$ & 1.046890 & 5489.75 & $4.48+$ \\
\hline 3 & Earth & $5.95522467 \times 10^{24}$ & $5.9736 \times 10^{24}$ & 0.996924 & 5479.43 & $0.31-$ \\
\hline 4 & Mars & $8.86381704 \times 10^{23}$ & $6.4185 \times 10^{23}$ & 1.380980 & 5401.97 & $27.59+$ \\
\hline 5 & Jupiter & $8.33471419 \times 10^{27}$ & $1.8986 \times 10^{27}$ & 4.389930 & 5445.40 & $77.22+$ \\
\hline 6 & Saturn & $4.98779142 \times 10^{27}$ & $5.6846 \times 10^{26}$ & 8.774220 & 5439.50 & $88.60+$ \\
\hline 7 & Uranus & $3.81282206 \times 10^{26}$ & $8.6832 \times 10^{25}$ & 4.391030 & 5451.63 & $77.23+$ \\
\hline 8 & Neptune & $3.48877925 \times 10^{26}$ & $1.0243 \times 10^{26}$ & 3.406010 & 5483.42 & $98.29+$ \\
\hline 9 & Pluto & $3.75718421 \times 10^{22}$ & $1.2500 \times 10^{22}$ & 3.005750 & 5256.18 & $66.73+$ \\
\hline
\end{tabular}

Table 4. Planetary mass calculation based on the distance of the aphelion

\begin{tabular}{|c|l|r|c|c|c|c|}
\hline No. & Planet & Calculated mass $(\mathrm{kg})$ & Current mass $(\mathrm{kg})$ & Calc./Curr. & Calc. density & Difference \% \\
\hline 1 & Mercury & $3.47771669 \times 10^{23}$ & $3.3020 \times 10^{23}$ & 1.053220 & 5717.37 & $5.05+$ \\
\hline 2 & Venus & $5.10955480 \times 10^{24}$ & $4.8685 \times 10^{24}$ & 1.049510 & 5503.52 & $4.72+$ \\
\hline 3 & Earth & $5.99302357 \times 10^{24}$ & $5.9736 \times 10^{24}$ & 1.003250 & 5514.21 & $0.32+$ \\
\hline 4 & Mars & $9.18077759 \times 10^{23}$ & $6.4185 \times 10^{23}$ & 1.430360 & 5595.14 & $30.09+$ \\
\hline 5 & Jupiter & $8.48954035 \times 10^{28}$ & $1.8986 \times 10^{27}$ & 4.471470 & 5546.55 & $77.64+$ \\
\hline 6 & Saturn & $5.09492608 \times 10^{27}$ & $5.6846 \times 10^{26}$ & 8.962680 & 5556.34 & $88.84+$ \\
\hline 7 & Uranus & $3.87844802 \times 10^{26}$ & $8.6832 \times 10^{25}$ & 4.466610 & 5545.46 & $77.61+$ \\
\hline 8 & Neptune & $3.50540081 \times 10^{26}$ & $1.0243 \times 10^{26}$ & 3.422240 & 5509.54 & $70.78+$ \\
\hline 9 & Pluto & $4.09382949 \times 10^{22}$ & $1.2500 \times 10^{22}$ & 3.275060 & 5727.14 & $69.47+$ \\
\hline
\end{tabular}

Table 5. Mass and distances calculated of Earth

\begin{tabular}{|l|r|c|r|r|l|}
\hline \multicolumn{1}{|c|}{ Magnitude } & Exp. & Calculated & Current & \multicolumn{2}{c|}{ Diff.(Cal.-Curr.) } \\
\hline Earth's mass & 24 & $5.9736 \times 10^{24} \mathrm{~kg}$. & $5.9736 \times 10^{24} \mathrm{~kg}$. & 0 & exact \\
\hline Perihelion & 22 & $1.46301102599553 \times 10^{11} \mathrm{~m}$. & $1.47098 \times 10^{11} \mathrm{~m}$. & -8 & thousandth \\
\hline Aphelion & 23 & $1.52983723851358 \times 10^{11} \mathrm{~m}$. & $1.52098 \times 10^{11} \mathrm{~m}$. & +9 & thousandth \\
\hline
\end{tabular}

The calculated mass of expressions (24) or (25) is a function of perihelion and aphelion previously calculated with the expressions (22) and (23). 
Table 6. Mass and distances calculated of Mercury

\begin{tabular}{|l|c|c|c|r|l|}
\hline \multicolumn{1}{|c|}{ Magnitude } & Exp. & Calculated & Current & \multicolumn{2}{c|}{ Diff. (Cal.Curr.) } \\
\hline Mercury's mass & 24 & $3.302 \times 10^{23} \mathrm{~kg}$. & $3.302 \times 10^{23} \mathrm{~kg}$. & 0 & exact \\
\hline Perihelion & 22 & $4.41646393275720 \times 10^{10} \mathrm{~m}$. & $4.600 \times 10^{10} \mathrm{~m}$. & -2 & tenths \\
\hline Aphelion & 23 & $7.70354595976312 \times 10^{10} \mathrm{~m}$. & $6.982 \times 10^{10} \mathrm{~m}$. & +7 & tenths \\
\hline
\end{tabular}

The calculated mass of expressions (24) or (25) is a function of perihelion and aphelion previously calculated with the expressions (22) and (23).

Table 7. Mercury mass calculated

\begin{tabular}{|l|c|c|c|c|l|}
\hline \multicolumn{1}{|c|}{ Magnitude } & Exp. & Calculated & Current & \multicolumn{2}{c|}{ Diff. (Cal./Curr.) } \\
\hline Mercury's mass & 24 & $3.34850063126456 \times 10^{23} \mathrm{~kg}$. & $3.302 \times 10^{23} \mathrm{~kg}$. & +5 & hundredth \\
\hline Perihelion & 22 & $4.31056763286010 \times 10^{10} \mathrm{~m}$. & $4.600 \times 10^{10} \mathrm{~m}$. & -3 & tenths \\
\hline Aphelion & 23 & $7.51883325166751 \times 10^{10} \mathrm{~m}$. & $6.982 \times 10^{10} \mathrm{~m}$. & +5 & tenths \\
\hline
\end{tabular}

Table 8. Velocities on the perihelion and aphelion

\begin{tabular}{|c|l|c|c|c|c|}
\hline & & \multicolumn{2}{|c|}{ Perihelion $\left(\right.$ radian. $\left.{ }^{-1}\right)$} & \multicolumn{2}{c|}{ Aphelion $\left(\right.$ radian. ${ }^{-1}$ ) } \\
\hline No. & Planet & Calculated & Current & Calculated & Current \\
\hline 1 & Mercury & $1.206208 \times 10^{-6}$ & $1.28217 \times 10^{-6}$ & $6.450437 \times 10^{-6}$ & $5.56574 \times 10^{-7}$ \\
\hline 2 & Venus & $3.665588 \times 10^{-7}$ & $3.28061 \times 10^{-6}$ & $3.592147 \times 10^{-7}$ & $3.19350 \times 10^{-7}$ \\
\hline 3 & Earth & $2.042451 \times 10^{-7}$ & $2.05917 \times 10^{-7}$ & $1.942570 \times 10^{-7}$ & $1.92573 \times 10^{-6}$ \\
\hline 4 & Mars & $2.179455 \times 10^{-7}$ & $1.28224 \times 10^{-7}$ & $1.645942 \times 10^{-7}$ & $8.81588 \times 10^{-8}$ \\
\hline 5 & Jupiter & $7.939009 \times 10^{-8}$ & $1.85275 \times 10^{-8}$ & $6.855537 \times 10^{-8}$ & $1.52335 \times 10^{-8}$ \\
\hline 6 & Saturn & $4.797991 \times 10^{-8}$ & $7.52652 \times 10^{-9}$ & $4.049352 \times 10^{-8}$ & $6.00198 \times 10^{-9}$ \\
\hline 7 & Uranus & $1.114037 \times 10^{-8}$ & $2.59366 \times 10^{-9}$ & $9.713305 \times 10^{-9}$ & $2.16073 \times 10^{-9}$ \\
\hline 8 & Neptune & $4.578268 \times 10^{-9}$ & $1.23750 \times 10^{-9}$ & $4.426204 \times 10^{-9}$ & $1.18134 \times 10^{-9}$ \\
\hline 9 & Pluto & $4.344501 \times 10^{-9}$ & $1.37486 \times 10^{-9}$ & $1.947124 \times 10^{-9}$ & $4.89709 \times 10^{-10}$ \\
\hline
\end{tabular}

Table 9. Calculated magnitudes for the perihelion

\begin{tabular}{|c|c|c|c|c|}
\hline No. & Planet & Calculated perihelion (meters) & Current perihelion (meters) & Calc./Curr. \\
\hline 1 & Mercury & $4.4164639327572105407714843750 \times 10^{10}$ & $4.6000000 \times 10^{10}$ & 0.96010 \\
\hline 2 & Venus & $1.0724674945444062805175781250 \times 10^{11}$ & $1.0748000 \times 10^{11}$ & 0.99782 \\
\hline 3 & Earth & $1.4630110259955313110351562499 \times 10^{11}$ & $1.4709829 \times 10^{11}$ & 0.99458 \\
\hline 4 & Mars & $2.0147469839847717285156250000 \times 10^{11}$ & $2.0666900 \times 10^{11}$ & 0.97486 \\
\hline 5 & Jupiter & $7.2949672436140454101562500000 \times 10^{11}$ & $7.4052000 \times 10^{11}$ & 0.98511 \\
\hline 6 & Saturn & $1.3263607213368405761718750000 \times 10^{12}$ & $1.3525500 \times 10^{12}$ & 0.98064 \\
\hline 7 & Uranus & $2.6989740586729760742187500000 \times 10^{12}$ & $2.7413000 \times 10^{12}$ & 0.98456 \\
\hline 8 & Neptune & $4.4175473769024208984375000000 \times 10^{12}$ & $4.4444500 \times 10^{12}$ & 0.99395 \\
\hline \multirow[t]{2}{*}{9} & Pluto & $4.2684334060828681640625000000 \times 10^{12}$ & $4.4368200 \times 10^{12}$ & 0.96205 \\
\hline & & & Average: & 0.98152 \\
\hline
\end{tabular}


Table 10. Calculated magnitudes for the aphelion

\begin{tabular}{|c|c|c|c|c|}
\hline No. & Planet & Calculated aphelion (meters) & Current aphelion (meters) & Calc./Curr. \\
\hline 1 & Mercury & $7.7035459597631301879882812499 \times 10^{10}$ & $6.9820000 \times 10^{10}$ & 1.103344 \\
\hline 2 & Venus & $1.0918824014965167236328124999 \times 10^{11}$ & $1.0894000 \times 10^{11}$ & 1.002278 \\
\hline 3 & Earth & $1.5298372385135861206054687500 \times 10^{11}$ & $1.5209823 \times 10^{11}$ & 1.005822 \\
\hline 4 & Mars & $2.5863680725410311889648437500 \times 10^{11}$ & $2.4920930 \times 10^{11}$ & 1.037830 \\
\hline 5 & Jupiter & $8.3119192084211376953125000000 \times 10^{11}$ & $8.1662000 \times 10^{11}$ & 1.017844 \\
\hline 6 & Saturn & $1.5423924170026977539062500000 \times 10^{12}$ & $1.5145000 \times 10^{12}$ & 1.018417 \\
\hline 7 & Uranus & $3.0484161974946367187500000000 \times 10^{12}$ & $3.0036200 \times 10^{12}$ & 1.014914 \\
\hline 8 & Neptune & $4.5564513550073632812500000000 \times 10^{12}$ & $4.5456700 \times 10^{12}$ & 1.002372 \\
\hline \multirow[t]{2}{*}{9} & Pluto & $8.4947099773799648437500000000 \times 10^{12}$ & $7.5759300 \times 10^{12}$ & 1.121276 \\
\hline & & & Average: & 1.036011 \\
\hline
\end{tabular}

Table 11. Calculation planetary mass based on corrected distances

\begin{tabular}{|r|l|c|c|c|c|r|}
\hline No. & Planet & Calculated mass $(\mathrm{kg})$ & Curr. Mass $(\mathrm{kg})$ & Calc./Curr. & Calc.density & Diff.\% \\
\hline 1 & Mercury & $3.30199999999999792381952 \times 10^{23}$ & $3.3020 \times 10^{23}$ & 1.00000 & 5428.49 & 0 \\
\hline 2 & Venus & $5.10302801772949604296294 \times 10^{24}$ & $4.8685 \times 10^{24}$ & 1.04817 & 5496.49 & $4.60+$ \\
\hline 3 & Earth & $5.97359999999999198887935 \times 10^{24}$ & $5.9736 \times 10^{24}$ & 1.00000 & 5496.34 & 0 \\
\hline 4 & Mars & $8.99722416792227815620608 \times 10^{23}$ & $6.4185 \times 10^{23}$ & 1.40176 & 5483.28 & $28.66+$ \\
\hline 5 & Jupiter & $8.40693555051561990208303 \times 10^{27}$ & $1.8986 \times 10^{27}$ & 4.42797 & 5492.58 & $77.42+$ \\
\hline 6 & Saturn & $5.04415396889423323979369 \times 10^{27}$ & $5.6846 \times 10^{26}$ & 8.87337 & 5500.97 & $88.73+$ \\
\hline 7 & Uranus & $3.84691957244456403761889 \times 10^{26}$ & $8.6832 \times 10^{25}$ & 4.43030 & 5500.38 & $77.43+$ \\
\hline 8 & Neptune & $3.50076217371727250086428 \times 10^{26}$ & $1.0243 \times 10^{26}$ & 3.41771 & 5502.25 & $70.74+$ \\
\hline 9 & Pluto & $3.85140130750609605263350 \times 10^{22}$ & $1.2500 \times 10^{22}$ & 3.08112 & 5387.99 & $67.54+$ \\
\hline & & & Average: & 3.18671 & 5476.53 & \\
\hline
\end{tabular}

Table 12. Gravitational constant " $G$ " and inertial forces

\begin{tabular}{|l|l|c|c|c|}
\hline \multirow{2}{*}{ Planet } & \multirow{2}{*}{ Satellite } & Gravitational constant "G" & \multicolumn{2}{c|}{ Repulsive action resulting in Newtons } \\
\cline { 3 - 5 } & & $(34)$ & Newton equation (35) & TCS equation (36) \\
\hline Earth & Moon & $6.67478 \times 10^{-11} \mathrm{~m}^{3} \mathrm{~kg}^{-1} \mathrm{~s}^{-2}$ & $5.45123 \times 10^{21} \mathrm{~N}$ & $6.49956 \times 10^{23} \mathrm{~N}$ \\
\hline Jupiter & Europa & $6.68047 \times 10^{-11} \mathrm{~m}^{3} \mathrm{~kg}^{-1} \mathrm{~s}^{-2}$ & $4.79616 \times 10^{22} \mathrm{~N}$ & $7.85295 \times 10^{23} \mathrm{~N}$ \\
\hline Uranus & Titania & $6.67400 \times 10^{-11} \mathrm{~m}^{3} \mathrm{~kg}^{-1} \mathrm{~s}^{-2}$ & $1.07374 \times 10^{20} \mathrm{~N}$ & $5.72044 \times 10^{20} \mathrm{~N}$ \\
\hline Pluto & Charon & $7.80841 \times 10^{-11} \mathrm{~m}^{3} \mathrm{~kg}^{-1} \mathrm{~s}^{-2}$ & $4.11600 \times 10^{18} \mathrm{~N}$ & $1.93405 \times 10^{19} \mathrm{~N}$ \\
\hline
\end{tabular}

Table 13. Data of the Moon

\begin{tabular}{|c|l|c|r|r|l|}
\hline Symbol & \multicolumn{1}{|c|}{ Data } & Exp. & \multicolumn{1}{c|}{ Calculated } & \multicolumn{1}{c|}{ Current } & Unit \\
\hline $\mathrm{m}_{\mathrm{L}}$ & Mass & $(24)$ or $(25)$ & $7.5181909708 \times 10^{22}$ & $7.349 \times 10^{22}$ & $\mathrm{~kg}$. \\
\hline$\rho_{\mathrm{L}}$ & Density & & 3418.2235748 & 3350. & $\mathrm{~kg} . \mathrm{m}^{-3}$. \\
\hline$\psi_{\mathrm{mL}}$ & Perihelion velocity & $(38)$ & $2.88439 \times 10^{-6}$ & $2.96174 \times 10^{-6}$ & $\mathrm{rad} . \mathrm{s}^{-1}$ \\
\hline$\psi_{\mathrm{ML}}$ & Aphelion velocity & $(38)$ & $2.44605 \times 10^{-6}$ & $2.37731 \times 10^{-6}$ & $\mathrm{rad} . \mathrm{s}^{-1}$ \\
\hline $\mathrm{d}_{\mathrm{mL}}$ & Perihelion distance & $(22)$ & $3.633 \times 10^{8}$ & $3.633 \times 10^{8}$ & $\mathrm{~m}$. \\
\hline $\mathrm{d}_{\mathrm{ML}}$ & Aphelion distance & $(23)$ & $4.055 \times 10^{8}$ & $4.055 \times 10^{8}$ & $\mathrm{~m}$. \\
\hline
\end{tabular}

Consider the expressions (22), (23), (24) and (25) to insert the drag coefficient field correction. Has been calculated the angular velocities with the distances current in the perihelion and aphelion. 


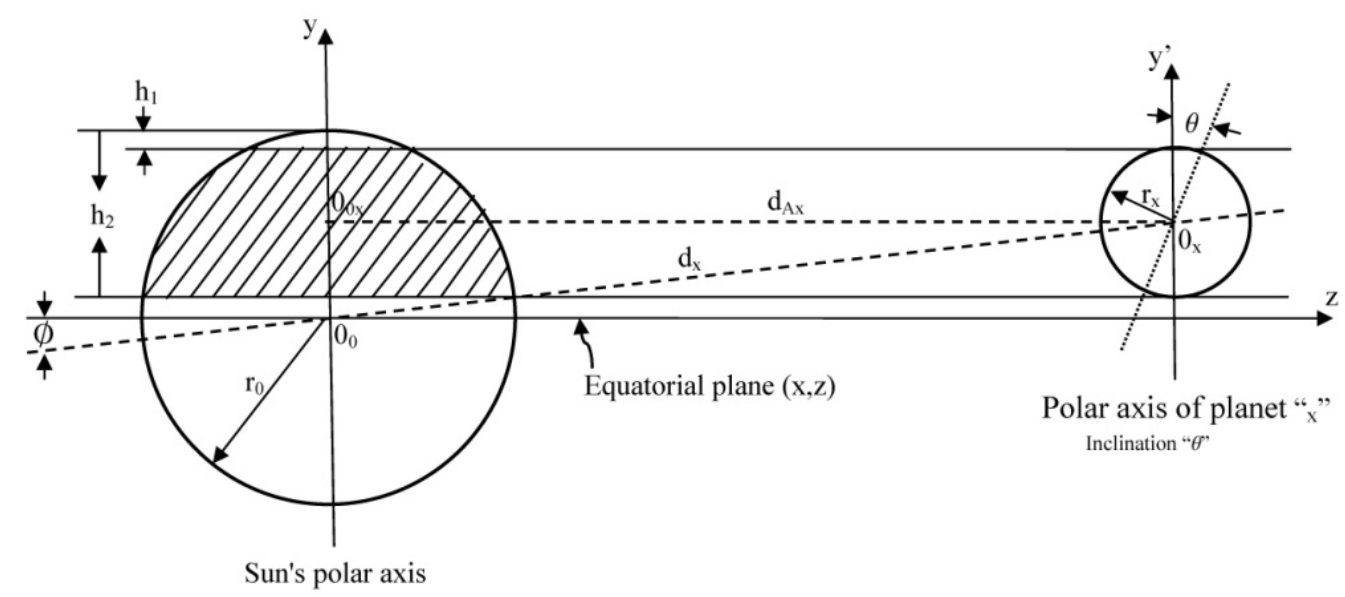

Figure 1. Reference data for the drag force of the Sun

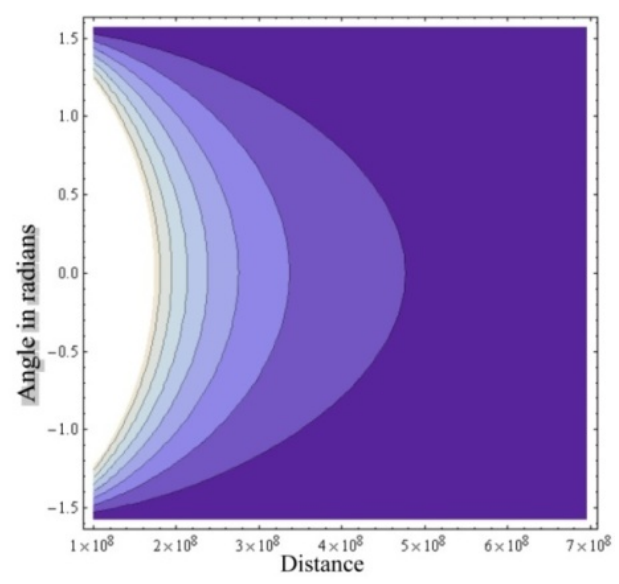

Figure 2. Equipotential field drag forces

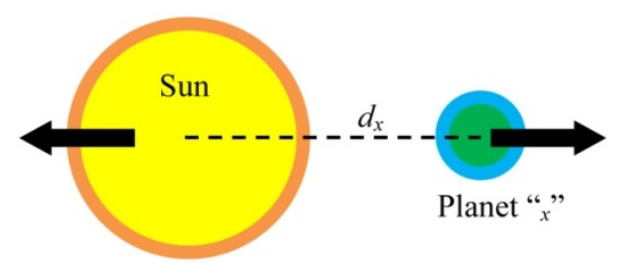

Figure 3. Electro-gravitational force

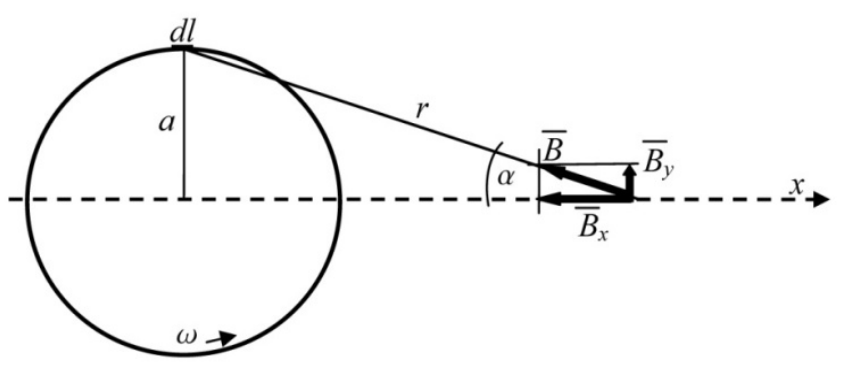

Figure 4. Magneto-gravitational vector 


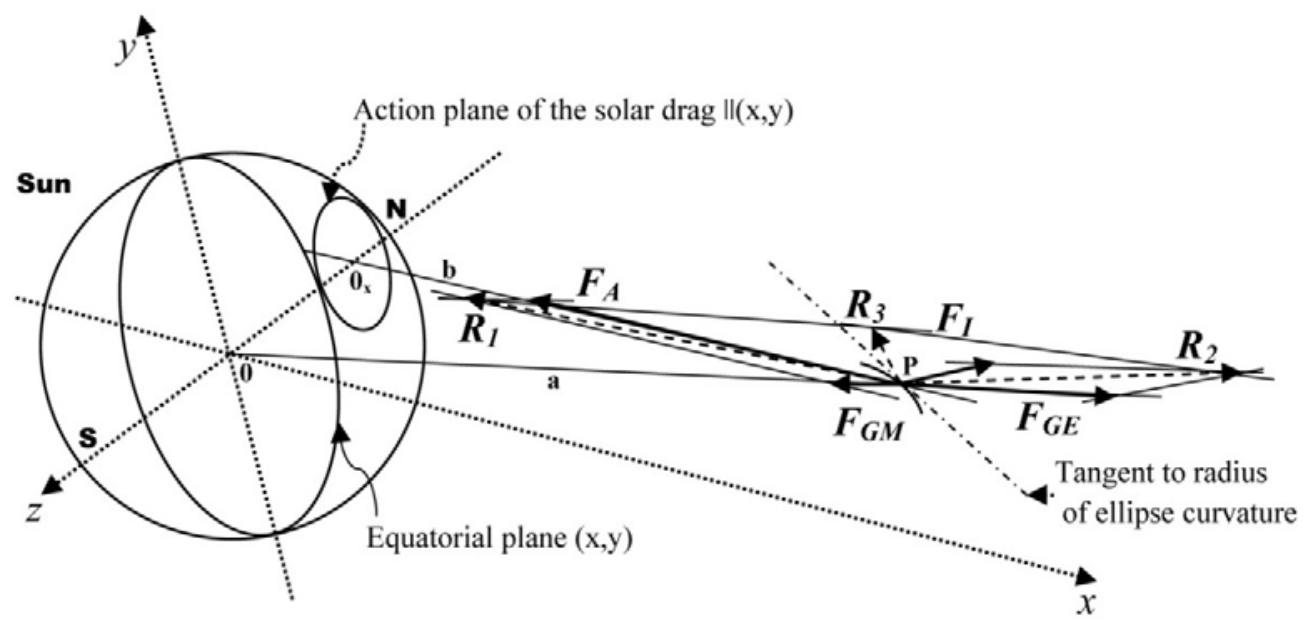

Figure 5. Diagram of action of the planetary gravitational forces

\section{Appendix}

$\underline{\text { Symbol }}$

Constant magnitude

$$
\begin{aligned}
k_{G E} & =2.88821919179004282377688826727 \times 10^{-10} \mathrm{~N} \cdot \mathrm{m}^{2} \cdot \mathrm{kg}^{-2} \\
k_{G M} & =3.57558690249434318757018569465 \times 10^{-44} \mathrm{~N} \cdot \mathrm{kg}^{-1} \\
Y_{P} & =2.53265203011334180831909179688 \times 10^{9} \quad \mathrm{~m}^{-1} \cdot \mathrm{s}^{-1}
\end{aligned}
$$

\begin{tabular}{|c|c|c|c|c|c|}
\hline Symbol & Magnitude & $\underline{\text { Symbol }}$ & Magnitude & $\underline{\text { Symbol }}$ & Magnitude \\
\hline & $365329 \times 10^{-6}$ & \multicolumn{2}{|c|}{$\mathrm{r}_{0}=6.96 \times 10^{8}$} & \multicolumn{2}{|c|}{$\mathrm{m}_{0}=1.9891 \times 10^{30}$} \\
\hline & 08. ; & \multicolumn{2}{|c|}{$\theta_{0}=7.25^{\circ}$} & & \\
\hline
\end{tabular}

0 - Sun (subscript: 0)

1 - Mercury (subscript: 1 , perihelion: ${ }_{\mathrm{m} 1}$, and aphelion: ${ }_{\mathrm{M} 1}$ )

$$
\begin{array}{rlrl}
\rho_{1} & =5427 . ; & \mathrm{m}_{1}=3.302 \times 10^{23} ; & \omega_{1}=1.2404614 \times 10^{-6} \\
\mathrm{v}_{\mathrm{M} 1}=38860 ; & & \phi_{1}=14.25^{\circ} ; \\
\mathrm{v}_{\mathrm{m} 1}=58980 . ; & \mathrm{r}_{1}=2.4397 \times 10^{6} ; & \theta_{1}=0 . .^{\circ} ; \\
\mathrm{v}_{1}=1 / 2\left(\mathrm{v}_{\mathrm{M} 1}+\mathrm{v}_{\mathrm{m} 1}\right) ; & \mathrm{d}_{\mathrm{m} 1}=4.6 \times 10^{10} ; & \mathrm{d}_{\mathrm{M} 1}=6.982 \times 10^{10} ; \\
\psi_{\mathrm{M} 1}=\mathrm{v}_{\mathrm{M} 1} / \mathrm{d}_{\mathrm{M} 1} ; & \mathrm{d}_{1}=\mathrm{v}_{1} \times 87.968 \times 864002 \pi ; & \\
\psi_{\mathrm{m} 1}=\mathrm{v}_{\mathrm{m} /} / \mathrm{d}_{\mathrm{m} 1} ; & \psi_{1}=\mathrm{v}_{1} / \mathrm{d}_{1} ; &
\end{array}
$$

$$
{ }_{\mathrm{m} 2} \text {, and aphelion: }{ }_{\mathrm{M} 2} \text { ) }
$$

$$
\begin{array}{rlrl}
\rho_{2} & =5243 . ; & \mathrm{m}_{2}=4.8685 \times 10^{24} ; & \omega_{2}=2.992420 \times 10^{-7} \\
\mathrm{v}_{\mathrm{M} 2}=34790 ; & & \phi_{2}=10.64^{\circ} ; \\
\mathrm{v}_{\mathrm{m} 2}=35260 . ; & \mathrm{r}_{2}=6.0518 \times 10^{6} ; & \theta_{2}=2.64^{\circ} ; \\
\mathrm{v}_{2}=1 / 2\left(\mathrm{v}_{\mathrm{M} 2}+\mathrm{v}_{\mathrm{m} 2}\right) ; & \mathrm{d}_{\mathrm{m} 2}=1.07480 \times 10^{11} ; & \mathrm{d}_{\mathrm{M} 2}=1.08940 \times 10^{11} ; \\
\psi_{\mathrm{M} 2}=\mathrm{v}_{\mathrm{M} 2} / \mathrm{d}_{\mathrm{M} 2} ; & \mathrm{d}_{2}=\mathrm{v}_{2} \times 224.695 \times 864002 \pi ; & \\
\psi_{\mathrm{m} 2}=\mathrm{v}_{\mathrm{m} 2} \mathrm{~d}_{\mathrm{m} 2} ; & \psi_{2}=\mathrm{v}_{2} / \mathrm{d}_{2} ; &
\end{array}
$$


3 - Earth (subscript: 3, perihelion: ${ }_{\mathrm{m} 3}$, and aphelion: ${ }_{\mathrm{M} 3}$ )

\begin{tabular}{|c|c|c|}
\hline$\rho_{3}=5515$ & $\mathrm{~m}_{3}=5.9736 \times 1024$ & $\omega_{3}=7.2921066 \times 10^{-5}$ \\
\hline $\mathrm{v}_{\mathrm{M} 3}=29290$. & $\mathrm{m}_{3^{*}}=6.04709 \times 10^{24}$ & $\phi_{3}=7.25^{\circ}$ \\
\hline $\mathrm{v}_{\mathrm{m} 3}=30290$ & $\mathrm{r}_{3}=6.3781 \times 10^{6}$ & $\theta_{3}=16.19^{\circ}$ \\
\hline $\mathrm{v}_{3}=1 / 2\left(\mathrm{v}_{\mathrm{M} 3}+\mathrm{v}_{\mathrm{m} 3}\right)$ & $\mathrm{d}_{\mathrm{m} 3}=1.4709829 \times 10^{11}$ & $\mathrm{~d}_{\mathrm{M} 3}=1.52098232 \times 10^{11} ;$ \\
\hline$\psi_{\mathrm{M} 3}=\mathrm{v}_{\mathrm{M} 3} / \mathrm{d}_{\mathrm{M} 3}$ & $\mathrm{~d}_{2}=\mathrm{v}_{2} \times 365.242 \times 86400 / 2 \pi$ & \\
\hline$\psi_{\mathrm{m} 3}=\mathrm{v}_{\mathrm{m} 3} / \mathrm{d}_{\mathrm{m} 3}$ & $\psi_{3}=\mathrm{v}_{3} / \mathrm{d}_{3}$ & \\
\hline
\end{tabular}

4 - Mars (subscript: 4, perihelion: ${ }_{\mathrm{m} 4}$, and aphelion: ${ }_{\mathrm{M} 4}$ )

$$
\begin{aligned}
& \rho_{4}=3933.5 \text {; } \\
& \mathrm{m}_{4}=6.4185 \times 10^{23} ; \\
& \mathrm{v}_{\mathrm{M} 4}=21970 \text {.; } \\
& \mathrm{m}_{4^{*}}=6.41850013 \times 10^{24} \text {; } \\
& \omega_{4}=7.08824 \times 10^{-5} \\
& \mathrm{v}_{\mathrm{m} 4}=26500 \text {. ; } \\
& \mathrm{r}_{4}=3.3962 \times 10^{6} \text {; } \\
& \phi_{4}=9.1^{\circ} \text {; } \\
& \mathrm{v}_{4}=1 / 2\left(\mathrm{v}_{\mathrm{M} 4}+\mathrm{v}_{\mathrm{m} 4}\right) ; \\
& \mathrm{d}_{\mathrm{m} 4}=2.06669 \times 10^{11} \text {; } \\
& \theta_{4}=25.19^{\circ} \text {; } \\
& \psi_{\mathrm{M} 4}=\mathrm{v}_{\mathrm{M} 4} / \mathrm{d}_{\mathrm{M} 4} \text {; } \\
& \mathrm{d}_{4}=\mathrm{V}_{4} \times 686.973 \times 86400 / 2 \pi \text {; } \\
& \psi_{\mathrm{m} 4}=\mathrm{v}_{\mathrm{m} 4} / \mathrm{d}_{\mathrm{m} 4} ; \\
& \psi_{4}=\mathrm{v}_{4} / \mathrm{d}_{4} \text {; } \\
& \mathrm{d}_{\mathrm{M} 4}=2.492093 \times 10^{11} \text {; }
\end{aligned}
$$

5 - Jupiter (subscript: 5, perihelion: ${ }_{\mathrm{m} 5}$, and aphelion: ${ }_{\mathrm{M} 5}$ )

$$
\begin{aligned}
& \rho_{5}=1326 \text {. } \quad \mathrm{m}_{5}=1.8986 \times 10^{27} ; \\
& \mathrm{v}_{\mathrm{M} 5}=12440 \text {.; } \\
& \mathrm{m}_{5^{*}}=1.89874055 \times 10^{27} \text {; } \\
& \omega_{5}=1.758518138 \times 10^{-4} \\
& \mathrm{v}_{\mathrm{m} 5}=13720 \text {. ; } \\
& \mathrm{r}_{5}=7.1492 \times 10^{7} \text {; } \\
& \phi_{5}=8.554^{\circ} \text {; } \\
& \mathrm{v}_{5}=1 / 2\left(\mathrm{v}_{\mathrm{M} 5}+\mathrm{v}_{\mathrm{m} 5}\right) \text {; } \\
& \mathrm{d}_{\mathrm{m} 5}=7.4052 \times 10^{11} \text {; } \\
& \theta_{5}=3.13^{\circ} \text {; }
\end{aligned}
$$

6 - Saturn (subscript: 6, perihelion: ${ }_{\mathrm{m} 6}$, and aphelion: ${ }_{\mathrm{M}}$ )

$$
\begin{aligned}
& \rho_{6}=687 \text {. ; } \\
& \mathrm{m}_{6}=5.6846 \times 10^{26} \text {; } \\
& \mathrm{v}_{\mathrm{M} 6}=9090 \text {. ; } \\
& \mathrm{m}_{6^{*}}=5.686006 \times 10^{26} \text {; } \\
& \omega_{6}=1.637884 \times 10^{-4} \\
& \mathrm{v}_{\mathrm{m} 6}=10180 \text {.; } \\
& \mathrm{r}_{6}=6.0268 \times 10^{7} \text {; } \\
& \mathrm{v}_{6}=1 / 2\left(\mathrm{v}_{\mathrm{M} 6}+\mathrm{v}_{\mathrm{m} 6}\right) \text {; } \\
& \mathrm{d}_{\mathrm{m} 6}=1.35255 \times 10^{12} \text {; } \\
& \phi_{6}=9.735^{\circ} \text {; } \\
& \psi_{\mathrm{M} 6}=\mathrm{v}_{\mathrm{M} 6} / \mathrm{d}_{\mathrm{M} 6} \text {; } \\
& \mathrm{d}_{6}=\mathrm{v}_{6} \times 10746.94 \times 86400 / 2 \pi \text {; } \\
& \psi_{\mathrm{m} 6}=\mathrm{v}_{\mathrm{m} 6} / \mathrm{d}_{\mathrm{m} 6} \text {; } \\
& \psi_{6}=\mathrm{v}_{6} / \mathrm{d}_{6} \text {; } \\
& \theta_{6}=26.73^{\circ} \text {; } \\
& \mathrm{d}_{\mathrm{M} 6}=1.5145 \times 10^{12} \text {; }
\end{aligned}
$$

7 - Uranus (subscript: 7, perihelion: ${ }_{\mathrm{m} 7}$, and aphelion: ${ }_{\mathrm{M} 7}$ )

$$
\begin{aligned}
& \rho_{7}=1270 \text {. ; } \\
& \mathrm{m}_{7}=8.6832 \times 10^{25} \text {; } \\
& \mathrm{v}_{\mathrm{M} 7}=6490 \text {. ; } \\
& \mathrm{v}_{\mathrm{m} 7}=7110 \text {. ; } \\
& \mathrm{m}_{7^{*}}=8.684112 \times 10^{25} \text {; } \\
& \mathrm{v}_{7}=1 / 2\left(\mathrm{v}_{\mathrm{M} 7}+\mathrm{v}_{\mathrm{m} 7}\right) ; \\
& \mathrm{r}_{7}=2.5559 \times 10^{7} \text {; } \\
& \omega_{7}=1.012372 \times 10^{-4} \\
& \phi_{7}=8.022^{\circ} \text {; } \\
& \theta_{7}=7.77^{\circ} \text {; } \\
& \mathrm{d}_{\mathrm{m} 7}=2.7413 \times 10^{12} \text {; } \\
& \mathrm{d}_{\mathrm{M} 7}=3.00362 \times 10^{12} \text {; }
\end{aligned}
$$

8 - Neptune (subscript: 8, perihelion: ${ }_{\mathrm{m} 8}$, and aphelion: ${ }_{\mathrm{M} 8}$ )

$$
\begin{aligned}
& \rho_{8}=1638 \text {.; } \\
& \mathrm{m}_{8}=1.0243 \times 10^{26} \text {; } \\
& \omega_{8}=1.08338 \times 10^{-4} \\
& \mathrm{v}_{\mathrm{M} 8}=5370 \text {. ; } \\
& \mathrm{m}_{8^{*}}=1.239219 \times 10^{26} \text {; } \\
& \phi_{8}=9.019^{\circ} \text {; } \\
& \mathrm{v}_{\mathrm{m} 8}=5500 \text {.; } \\
& \mathrm{r}_{8}=2.4765352 \times 10^{7} \text {; } \\
& \theta_{8}=28.32^{\circ} \text {; } \\
& \mathrm{v}_{8}=1 / 2\left(\mathrm{v}_{\mathrm{M} 8}+\mathrm{v}_{\mathrm{m} 8}\right) \text {; } \\
& \mathrm{d}_{\mathrm{m} 8}=4.44445 \times 10^{12} \text {; } \\
& \mathrm{d}_{\mathrm{M} 8}=4.54567 \times 10^{12} \text {; }
\end{aligned}
$$


9 - Pluto (subscript: 9, perihelion: ${ }_{\mathrm{m} 9}$, and aphelion: ${ }_{\mathrm{m} 9}$ )

$$
\begin{array}{rlrl}
\rho_{9} & =1750 . ; & \mathrm{m}_{9} & =1.25 \times 10^{22} ; \\
\mathrm{v}_{\mathrm{M} 9} & =3710 . ; & \mathrm{m}_{9 *}=1.412 \times 10^{22} ; & \omega_{9}=0.4618 \\
\mathrm{v}_{\mathrm{m} 9}=6100 . ; & \mathrm{r}_{9}=1.195 \times 10^{6} ; & \theta_{9}=24.41^{\circ} ; \\
\mathrm{v}_{9}=1 / 2\left(\mathrm{v}_{\mathrm{M} 9}+\mathrm{v}_{\mathrm{m} 9}\right) ; & \mathrm{d}_{\mathrm{m} 9}=4.43682 \times 10^{\circ} ; & \mathrm{d}_{\mathrm{M} 9}=7.57593 \times 10^{12} ; \\
\psi_{\mathrm{M} 9}=\mathrm{v}_{\mathrm{M} 9} / \mathrm{d}_{\mathrm{M} 9} ; & \mathrm{d}_{9}=\mathrm{v}_{9} \times 90588 . \times 864002 \pi ; & \\
\psi_{\mathrm{m} 9}=\mathrm{v}_{\mathrm{m} 9} 9 \mathrm{~d}_{\mathrm{m} 9} ; & \psi_{9}=\mathrm{v}_{9} / \mathrm{d}_{9} ; &
\end{array}
$$

\section{Satellites}

M - Moon-Earth (subscript: M, perihelion: ${ }_{\mathrm{mM}}$, and aphelion: ${ }_{\mathrm{MM}}$ )

$$
\begin{aligned}
\rho_{\mathrm{M}}=3350 . ; & \mathrm{m}_{\mathrm{M}}=7.349 \times 10^{22} ; & \omega_{\mathrm{M}}=7.2921066 \times 10^{-5} \\
\mathrm{v}_{\mathrm{MM}}=964 . ; & \mathrm{r}_{\mathrm{M}}=1.7381 \times 10^{6} ; & \phi_{\mathrm{M}}=5.145^{\circ} ; \\
\mathrm{v}_{\mathrm{mM}}=1076 . ; & & \theta_{\mathrm{M}}=6.68^{\circ} ; \\
\mathrm{v}_{\mathrm{M}}=1 / 2\left(\mathrm{v}_{\mathrm{MM}}+\mathrm{v}_{\mathrm{mM}}\right) ; & \mathrm{d}_{\mathrm{mM}}=3.633 \times 10^{8} ; & \mathrm{d}_{\mathrm{MM}}=4.055 \times 10^{8} ; \\
\psi_{\mathrm{MM}}=\mathrm{v}_{\mathrm{MM}} / \mathrm{d}_{\mathrm{MM}} ; & \mathrm{d}_{\mathrm{M}}=\mathrm{v}_{\mathrm{M}} \times 1 . \times 864002 \pi ; & \\
\psi_{\mathrm{mM}}=\mathrm{v}_{\mathrm{mM}} / \mathrm{d}_{\mathrm{mM}} ; & \psi_{\mathrm{M}}=\mathrm{v}_{\mathrm{M}} / \mathrm{d}_{\mathrm{M}} ; &
\end{aligned}
$$

E - Europa-Jupiter (subscript: E, perihelion: ${ }_{\mathrm{mE}}$, and aphelion: ${ }_{\mathrm{ME}}$ )

$$
\begin{aligned}
\rho_{\mathrm{E}}=3010 . ; & \mathrm{m}_{\mathrm{E}}=4.8 \times 10^{22} ; & \omega_{\mathrm{E}}=2.048509 \times 10^{-5} \\
\mathrm{v}_{\mathrm{ME}}=13700 ; ; & \mathrm{r}_{\mathrm{E}}=1.5608 \times 10^{6} ; & \phi_{\mathrm{E}}=0 .^{\circ} ; \\
\mathrm{v}_{\mathrm{mE}}=13800 . ; & & \theta_{\mathrm{E}}=0 .^{\circ} ; \\
\mathrm{v}_{\mathrm{E}}=1 / 2\left(\mathrm{v}_{\mathrm{ME}}+\mathrm{v}_{\mathrm{mE}}\right) ; & \mathrm{d}_{\mathrm{mE}}=6.64862 \times 10^{8} ; & \mathrm{d}_{\mathrm{ME}}=6.76938 \times 10^{8} ; \\
\psi_{\mathrm{ME}}=\mathrm{v}_{\mathrm{ME}} / \mathrm{d}_{\mathrm{ME}} ; & \mathrm{d}_{\mathrm{E}}=\mathrm{v}_{\mathrm{E}} \times 1 . \times 86400 / 2 \pi ; & \\
\psi_{\mathrm{mE}}=\mathrm{v}_{\mathrm{m} 2} / \mathrm{d}_{\mathrm{mE}} ; & \psi_{\mathrm{E}}=\mathrm{v}_{\mathrm{E}} / \mathrm{d}_{\mathrm{E}} ; &
\end{aligned}
$$

T - Titania-Uranus (subscript: T, perihelion: ${ }_{\mathrm{mT}}$, and aphelion: ${ }_{\mathrm{MT}}$ )

$$
\begin{array}{rlrl}
\rho_{\mathrm{T}} & =1711 . ; & \mathrm{m}_{\mathrm{T}}=3.527 \times 10^{21} ; & \omega_{\mathrm{T}}=1 . \times 10^{-7} \\
\mathrm{v}_{\mathrm{MT}}=3640.5 ; & \mathrm{r}_{\mathrm{T}}=7.88971 \times 10^{5} ; & \phi_{\mathrm{T}}=0 .{ }^{\circ} ; \\
\mathrm{v}_{\mathrm{mT}}=3648.52 ; & & \theta_{\mathrm{T}}=0 . .^{\circ} ; \\
\mathrm{v}_{\mathrm{T}}=1 / 2\left(\mathrm{v}_{\mathrm{MT}}+\mathrm{v}_{\mathrm{mT}}\right) ; & \mathrm{d}_{\mathrm{mT}}=4.35821 \times 10^{8} ; & \mathrm{d}_{\mathrm{MT}}=4.3678 \times 10^{8} ; \\
\psi_{\mathrm{MT}}=\mathrm{v}_{\mathrm{MT}} / \mathrm{d}_{\mathrm{MT}} ; & \mathrm{d}_{\mathrm{T}}=\mathrm{v}_{\mathrm{T}} \times 8.705872 \times 864002 \pi ; & \\
\psi_{\mathrm{mT}}=\mathrm{v}_{\mathrm{m}} \mathrm{d} \mathrm{d}_{\mathrm{mT}} ; & \psi_{\mathrm{T}}=\mathrm{v}_{\mathrm{T}} / \mathrm{d}_{\mathrm{T}} ; &
\end{array}
$$

C - Charon-Pluto (subscript: C, perihelion: ${ }_{\mathrm{mC}}$, and aphelion: , $_{\mathrm{MC}}$ )

$$
\begin{array}{rlrl}
\rho_{\mathrm{C}} & =1850 . ; & \mathrm{m}_{\mathrm{C}}=1.62 \times 10^{21} ; & \omega_{\mathrm{C}}=1.13855 \times 10^{-5} \\
\mathrm{v}_{\mathrm{MC}}=223.1558 ; & \mathrm{r}_{\mathrm{C}}=5.93 \times 10^{5} ; & \phi_{\mathrm{C}}=0 .^{\circ} ; \\
\mathrm{v}_{\mathrm{mC}}=223.1558 ; & & \theta_{\mathrm{C}}=0 . .^{\circ} ; \\
\mathrm{v}_{\mathrm{C}}=1 / 2\left(\mathrm{v}_{\mathrm{MC}}+\mathrm{v}_{\mathrm{mC}}\right) ; & \mathrm{d}_{\mathrm{mC}}=1.96 \times 10^{7} ; & \mathrm{d}_{\mathrm{MC}}=1.96 \times 10^{7} ; \\
\psi_{\mathrm{MC}}=\mathrm{v}_{\mathrm{MC}} / \mathrm{d}_{\mathrm{MC}} ; & \mathrm{d}_{\mathrm{C}}=\mathrm{v}_{\mathrm{C}} \times 6.38725 \times 864002 \pi ; & \\
\psi_{\mathrm{mC}}=\mathrm{v}_{\mathrm{m}} / \mathrm{d}_{\mathrm{mC}} ; & \psi_{\mathrm{C}}=\mathrm{v}_{\mathrm{C}} \mathrm{d}_{\mathrm{C}} ; &
\end{array}
$$

Note 1 (subscript *): The sum of the estimated mass of the planet today most of its satellites. Equation (42) is to calculate the average radius of the planet with mass plus the mass of its satellites.

$$
r_{x^{*}}=\sqrt[3]{\frac{3 m_{x^{*}}}{4 \pi \rho_{x}}}
$$

Note 2 (dimension in each variable): $\rho_{x}\left(\mathrm{~kg} \cdot \mathrm{m}^{-3}\right) ; \mathrm{m}_{x}(\mathrm{~kg}) ; \omega_{x}$ and $\psi_{x}$ (radians. $\left.\mathrm{s}^{-1}\right) ; \mathrm{v}_{x}\left(\mathrm{~m} \cdot \mathrm{s}^{-1}\right) ; \mathrm{r}_{x}$ and $\mathrm{d}_{x}($ meters); and $\phi_{x}$ and $\theta_{x}$ (in degrees). 